\title{
ON THE EVOLUTION OF ANGULAR MOMENTUM, MAGNETIC ACTIVITY AND MASS LOSS RATE OF LATE TYPE MAIN SEQUENCE STARS
}

\author{
J. Bohigas \\ Instituto de Astronomía, Universidad Nacional Autónoma de México, México. \\ Received July 21 2019; accepted February 132020
}

\begin{abstract}
With the best data, I find that nearly all 0.5 to $1.2 M_{\odot}$ main sequence stars converge to a single rotational mass-dependent sequence after $750 \mathrm{Myr}$; when $\mathrm{M}>0.8 M_{\odot}$, most of them converge in $\approx 120 \mathrm{Myr}$. If stars rotate as rigid bodies, most have angular momenta within clear bounds. The lower bound defines a terminal main sequence rotational isochrone, the upper one coincides with slow rotators from the Pleiades; stars from Praesepe delineate a third one. Mass dependent exponential relationships between angular momentum and age are determined. Age estimates based on the angular momentum are acceptable for stars older than $750 \mathrm{Myr}$ and with $\mathrm{M}>0.6-0.7 M_{\odot}$. The Rossby number indicates that the Parker dynamo may cease early on in stars with $\mathrm{M} / M_{\odot} \geq 1.1$. An empirical formula and a model for the torque, and a relation between rotational period and magnetic field, lead to a formula for the evolution of the mass loss rate; the present solar rate is near a minimum and was about five times larger when life on Earth started.
\end{abstract}

\section{RESUMEN}

Con los mejores datos, encuentro que después de $750 \mathrm{Ma}$, casi todas las estrellas de 0.5 a $1.2 M_{\odot}$ de la secuencia principal convergen a una secuencia rotacional dependiente de la masa. Si M $>0.8 M_{\odot}$, la mayoría converge en $\approx 120 \mathrm{Ma}$. Si rotan como cuerpo rígido, casi todas tienen momentos angulares entre límites bien definidos. El inferior define la isocrona rotacional terminal, el superior coincide con rotores lentos de las Pléyades; las estrellas del Pesebre delinean un tercer límite. Se obtienen relaciones exponenciales entre momento angular y edad. Las edades basadas en el momento angular son aceptables para estrellas de edad mayor que 750 Ma y masa mayor que $0.6-0.7 M_{\odot}$. El número de Rossby indica que el dínamo de Parker se interrumpe tempranamente si $\mathrm{M} / M_{\odot} \geq 1.1$. Una fórmula empírica y un modelo para la torca, y una relación entre periodo rotacional y campo magnético conducen a una ecuación para la tasa de pérdida de masa; la tasa solar actual está cerca del mínimo y era cinco veces mayor cuando empezó la vida en la Tierra.

Key Words: stars: evolution — stars: late-type — stars: magnetic fields — stars: mass loss - stars: rotation

\section{INTRODUCTION}

There is a transition from rapidly to slowly rotating main sequence stars when the mass is less than $\approx 1.2$ to $1.3 M_{\odot}$ (Struve \& Elvey, 1931; Herbig \& Spalding, 1955; Slettebak, 1955; Treanor, 1960). Concurrently, chromospheric and coronal activity step up in the lower mass range (Wilson 1966; Pallavicini et al. 1981). In the Sun, chromospherically active regions and magnetic fields evolve in parallel (Bumba
\& Howard 1965) and X-ray images show that there is a relationship between magnetic fields and the hot corona (Vaiana et al. 1981). These connections lead to the notion that magnetic energy is the main source of chromospheric and coronal heating (e.g., Parker 1988; Cranmer \& Saar 2011; van Ballegooijen, Asgari-Targhi \& Berger, 2014). In turn, the highly pressurized hot corona expands to produce a stellar wind (Parker 1958; Cranmer et al. 2007; 
Suzuki et al. 2013; van der Holst et al. 2014; Usmanov et al. 2018).

Noting that low mass stars have convective envelopes, Schatzman (1962) reasoned that the magnetic fields generated by dynamo processes in a spinning star with a convective envelope (the $\alpha-\Omega$ mechanism, Parker 1955), compel the stellar wind to rotate with the star even at very large distances, where it carries away their angular momentum. Schatzman predicted a secular decrease in the angular momentum of these stars, and this was confirmed when Kraft (1967) compared the average surface values of the rotational velocity of $1.2 M_{\odot}$ stars from the Pleiades and Hyades open clusters. All these associations were explicitly identified by Skumanich (1972), who argued that Ca II emission, angular velocity and magnetic field strength are proportional to each other in this kind of stars, and predicted that these quantities decay as the inverse square root of time, inspiring the idea of using rotation and stellar activity as timekeeping devices for low mass stars.

Thus, there is a feedback loop between rotation and magnetic field, leading to angular frequency, field strength, activity and wind power decreasing with age if no other factors are involved. This feedback is also observed in fully convective stars (McQuillan, Agrain \& Mazeh 2013; Newton et al. 2018), where the magnetic field can not be produced by the $\alpha-\Omega$ mechanism. It has been submitted that a large density of small scale magnetic fields induced by a turbulent velocity field (Durney, De Young \& Roxburgh 1993), is even capable of, for instance, maintaining a hot coronal plasma (and a stellar wind) in M9 V type stars (Robrade \& Schmitt, 2009). An important point is that the $\mathrm{X}$-ray and chromospheric to bolometric luminosity ratios reach a maximum constant value when the rotational period is less than $\approx 2$ days (Soderblom et al. 1993; Pizzolato et al. 2003). In non-fully convective stars this is connected to a saturation value close to $4 \mathrm{kG}$ for the magnetic field strength (Reiners et al. 2014), but significantly larger field strengths have been measured in fully convective M dwarfs (Shulyak et al. 2017 and 2019). This has been associated to the dominance of axisymmetric poloidal and nearly dipolar magnetic fields in this type of stars, as opposed to the prevalence of multipolar fields in dwarf stars with a convective envelope and a radiative interior (Morin et al. 2010).

Using the equations of magnetohydrodynamics, Schatzman's (1962) idea has been linked to different versions of stellar wind theories and various possibilities for the structure of the coronal magnetic field in a rotating star (e.g., Weber \& Davis, 1967; Mestel 1968, 1984; Mestel \& Spruit, 1987; Réville et al. 2015). But due to the complex nature of angular momentum evolution of low mass main sequence stars, most theoretical work has been based on heuristic arguments, in order to find manageable equations for a mass and time dependent torque. These semi-empirical prescriptions make assumptions on the properties of the stellar wind and magnetic field, and their relation with the angular velocity and other observable stellar parameters, such as the Rossby number or the filling factor of the magnetized surface. Assumptions have changed in step with our understanding of the theoretical and observational properties of these stars, but the final equations for the torque still involve one or more calibration constants, as well as loose power law relations with the mass, radius, mass loss rate and angular velocity (e.g., Bohigas et al. 1986; Kawaler, 1988; Reiners \& Mohanty, 2012; Matt et al. 2015; Gallett \& Bouvier, 2015; Johnstone et al. 2015a).

Since the stellar angular momentum is removed from the atmosphere, it was initially believed that the radiative core would be spinning much faster than the convective envelope. Brown et al. (1989) established that this is not the case in the Sun, and we now know that the radial average of its rotational velocity is roughly constant at least down to $0.15 R_{\odot}$ (Kozennik \& Eff-Darwich, 2012; Eff-Darwich \& Korzennik, 2013). Evidence of flat differential rotation in solar type stars has been produced using asteroseismological techniques (Benomar et al. 2015; Nielsen et al. 2017). All these implies that there are efficient transport mechanisms of angular momentum from the radiative core to the convective envelope (see recent review in Aerts, Mathis \& Rogers 2019). Currently, models for angular momentum transport include an heuristic approximation where the transferred amount is just enough to maintain equilibrium at the core-envelope interface (MacGregor \& Brenner 1991; Gallet \& Bouvier 2015), braking the inner core by a pre-existing poloidal magnetic field (Mestel \& Weiss 1987; Charbonneau \& MacGregor 1993; Dennisenkov 2010), internal gravity waves generated at the convective envelope (Charbonnel \& Talon 2005; Charbonnel et al. 2013) and - without being able to simultaneously reproduce the surface period, rotational profile and lithium abundance of the Sun - internal transport of angular momentum by meridional circulation (Denisenkov 2010; Charbonnel et al. 2013; Amard et al. 2016).

Obviously, the angular momentum transfer rate depends on the torque running at the convective en- 
velope and combinations of rotational braking and angular momentum transport models have been designed by Denisenkov et al. (2010), Gallet \& Bouvier (2013 and 2015) and Amard et al. (2016). When dealing with close binary systems, a tidal synchronization torque must be included (Johnstone et al. 2019). And during the pre-main sequence phase, angular momentum transfer between the central star and the accretion disk can have a central role.

Setting "initial" conditions has not been easy. The existence of a wide spectrum of rotational periods in nearly equal mass stars from young clusters - such as $\mathrm{h}$ and $\alpha$ Persei, NGC 2547 and Pleiades (Stauffer et al. 1984 and 1985; Irwin et al. 2008; Moraux et al. 2013) - implies that initial conditions are not unique. Even when they end up having the same mass, members of the same cluster are not born at the same time, and with identical rotation rates and physical structures (e.g., Henderson \& Stassun 2012). Furthermore, Bouvier et al. (1993) found that the mean rotational period is significantly longer in classical T Tauri stars (CTTS) than in weak line T Tauri stars (WTTS). Noting that accretion disks and powerful winds are detected in CTTS, but not in WTTS, they explained this difference arguing that WTTS spin up as they contract without loosing angular momentum, whereas CTTS do not spin up as much (if at all) since angular momentum is still being removed by a powerful wind that is thought to be created by magnetic coupling between the central star and the accretion disk (Koenigl 1991; Collier Cameron \& Campbell 1993; Collier Cameron, Campbell \& Quaintrell 1995). Observations indicate that the rotational period is roughly constant during the disk locking phase (Rebull et al. 2004; Gallet \& Bouvier 2013). Longer periods and disk locking times are associated to slow rotators: for a solar mass star, around 8 days and 9 Myr vs. 1.4 days and 2 Myr for fast rotators (Gallet \& Bouvier 2015).

Angular momentum losses are greatly reduced after star-disk decoupling. The star spins up as it contracts and the rotational velocity reaches it's largest value when the star arrives at the ZAMS - after 40 and $200 \mathrm{Myr}$ for a 1.2 and $0.6 M_{\odot}$ star - and its internal structure is stabilized. Thereon, the rotational history is essentially - but not exclusively determined by the magnetic braking mechanism first described by Schatzman (1962).

Over the last two decades, this field of research has been greatly favored by vast improvements in observational procedures and methods of analysis focused on measuring the rotational period, parallax and magnitudes of stars. These were developed by research groups engaged in one particular field of stars or connected to long term and/or wide field campaigns, such as the Two Micron All Sky Survey $(2 M A S S)$, the European Space Mission satellite Gaia and NASA's Kepler satellite Mission. At least equally important has been the unfolding of exciting ideas and techniques - asteroseismology, Zeeman splitting and Doppler imaging, and astrospheric absorption - with which we are beginning to know the internal rotational structure, the superficial magnetic field intensity and geometry, and the mass loss rate of late type main sequence stars. Lastly, the connection with basic stellar parameters - such as mass, radio, temperature, convective turnover time and moment of inertia - has been made possible thanks to evolutionary models that are regularly being updated (i.e., VandenBerg et al. 2006; Feiden et al. 2011; Baraffe et al. 2015; Choi et al. 2016; Marigo et al. 2017; Spada et al. 2017).

This paper collects a vast amount of rotational, photometric and parallax data, to re-analyze the angular momentum history of $\approx 0.5-1.2 M_{\odot}$ main sequence stars, with an emphasis on those that are at least as old as the Pleiades cluster. All rotational periods, apparent magnitudes and any other observed parameter used to analyze some of these questions, are taken from the literature. In order to have a data set as uniform as possible, all infrared $J H K$ magnitudes were collected from the $2 M A S S$ project, and visual magnitudes were derived from the Gaia DR2 G, BP and RP band magnitudes. Likewise, only Gaia DR2 parallaxes have been used. To minimize uncertainties and improve data set quality, stars with additional causes of variability, poorly determined period and photometry, and uncertain parallax, were excluded from the analysis. Rotational periods are taken from several sources, but not all of them report error estimates. It is worth noting that discrepancies found in some rotational periods measured by more than one research group can be much larger than the reported errors. Therefore, rotational periods are the least reliable element in this compilation, which contains one of the largest and better selected data required to analyze the evolution of angular momentum of $\approx 0.5$ to $1.2 M_{\odot}$ main sequence stars. A general discussion of data sources is presented in $\S 2.1$, and the criteria that were used to select the best possible information are discussed in $\S 2.2$. Procedures used to obtain absolute magnitudes and stellar parameters are described in subsections $\S 2.3$ and $\S 2.4$. 
The distribution of rotational periods as a function of mass and time is presented and discussed in $§ 3.1$. Assuming rigid body rotation, the behavior of angular momentum as a function of mass and age is discussed in subsection $§ 3.2$. This is not the usual approach used to study the rotational history of low mass main sequence stars but, as noticed by other authors (Johnstone et al. 2015a), the observational evidence we now have on their inner rotational structure, as well as models for angular momentum redistribution within them, indicate that this is not an unlikely hypothesis for stars older than a few hundred million years, or maybe less. Formulae relating mass and angular momentum in three rotational isochrones are included in this subsection. These are used to introduce two possible approximations for the evolution of angular momentum in main sequence stars having a mass between 0.5 and $1.2 M_{\odot}$, using a $0.1 M_{\odot}$ bin to downsize variations due to mass differences. The special circumstances and time periods under which it is possible to obtain an age estimate directly from the rotational period are discussed in $§ 3.3$. Magnetic activity as a function of mass and time is analyzed in $§ 3.4$. Another important bonus of the rigid body rotation hypothesis is that it leads directly to simple empirical formulae for the torque as a function of mass and time, without having to use shortcuts to put together relationships between, among other things, mass, magnetic field, mass loss rate and angular velocity (subsection §3.5). To some extent, it turns the problem around. For instance, in combination with known connections between age and the magnetic intensity at the stellar surface, and a simple model for the magnetic field source, these formulae lead to a clear-cut procedure to assess the mass loss rate (subsection §3.6), the least known important trait of low mass main sequence stars. The most significant results of this paper are summed up in the final section.

\section{DATA}

Star spots and plages transiting the stellar surface can produce a quasi-periodic modulation of light intensity that is correlated with a stellar rotation rate, $\Omega$. Supported by long term photometric observations, readily available rotational data from a vast number of field and cluster late type main sequence stars have been produced by several research groups over the last two decades. These groups carried out meticulous time series analyses of light curves in order to determine rotational periods, in most cases using different formulations of the LombScargle method (Lomb 1976; Scargle 1982, 1989).
But notice that there is an inherent uncertainty in the physical connotation of the computed value of the rotational period at the stellar surface, since the correlation between light intensity and period is not straight forward or unambiguous. The position, lifetime and extension of star spots, plages and other superficial features, are conditioned by the latitudinal rotational shear, which is caused by rotating stellar convection transporting angular momentum from the pole to the equator, and thermal energy in the opposite direction (Busse 1970; Durney 1970; Kitchatinov \& Rüdiger 2005; Küker \& Rüdiger 2008). Differential rotation tends to be more pronounced in stars with lower rotational periods (Henry et al. 1995), higher effective temperatures (Barnes et al. 2005) and shallower convective regions (Küker \& Rüdiger 2008). Thus, the relation between these inconstant superficial features and rotational period depends on stellar mass, metallicity and age, increasingly so in spectral types where differential rotation is more pronounced and variable, and when stars are close to the beginning or the end of main sequence. As a matter of fact, Epstein \& Pinsonneault (2014) noticed that latitudinal surface differential rotation introduces significant ambiguities into rotation period measurements, leading to a $\approx 2000$ Myr uncertainty in rotation-based age estimates. All these should not be forgotten when the mass and time dependence of the rotational evolution of late type main sequence stars is considered.

\subsection{Data Sources}

The rotational periods of field and open cluster stars contained in 28 publications were collected in order to investigate the evolution of angular momentum during the main sequence. Twenty three of these papers are dedicated to 17 open star clusters in the Milky Way. The open cluster sample was put together with the intention of covering the largest possible range of ages after the termination of the disk locking phase. With an estimated age of 13 and 3500 Myr, h Persei and M 67 are the youngest and oldest open clusters inspected in this work. The central positions (epoch 2000.0), angular radii $(\Theta)$, parallaxes, ages, color excesses $(E(B-V))$ and metallicities $([\mathrm{Fe} / \mathrm{H}])$ of these clusters are laid out in Table 1 (references in Appendix A). Some of these numbers were used in data selection and extinction correction, $\S 2.2$ and $\S 2.3$. The cluster age, metallicity and assumed initial helium abundance $\left(Y_{0}\right)$ are the input parameters for the evolutionary tracks used to derive the physical properties of cluster stars from their visual and infrared colors, $§ 2.4$. 
TABLE 1

CLUSTER PARAMETERS*

\begin{tabular}{lrrrrrrrl}
\hline Cluster & \multicolumn{1}{c}{ RA } & \multicolumn{1}{c}{$\begin{array}{c}\text { DEC } \\
\text { deg }\end{array}$} & \multicolumn{1}{c}{$\begin{array}{c}\text { deg } \\
\text { deg }\end{array}$} & $\begin{array}{c}\text { Parallax } \\
\text { mas }\end{array}$ & \multicolumn{1}{c}{$\begin{array}{c}\text { Age } \\
\text { Myr }\end{array}$} & $\begin{array}{c}E(B-V) \\
-\end{array}$ & \multicolumn{1}{c}{$[F e / H]$} & $\mathrm{Y}_{0}$ \\
\hline h Persei & 34.770 & 57.150 & 1.75 & $0.547 \pm 0.122$ & $13 \pm 1$ & $0.515 \pm 0.035$ & 0.00 & 0.28 \\
IC 4665 & 266.498 & 5.565 & 1.00 & $2.892 \pm 0.003$ & $40 \pm 10$ & $0.177 \pm 0.003$ & $-0.06 \pm 0.10$ & $0.27 \pm 0.01$ \\
NGC 2547 & 122.565 & -49.050 & 0.30 & $2.544 \pm 0.002$ & $40 \pm 10$ & $0.050 \pm 0.010$ & $-0.14 \pm 0.10$ & $0.27 \pm 0.01$ \\
Blanco 1 & 1.029 & 29.833 & 2.90 & $4.194 \pm 0.041$ & $100 \pm 30$ & $0.010 \pm 0.000$ & $0.03 \pm 0.07$ & $0.28 \pm 0.01$ \\
Pleiades & 56.300 & 24.120 & 6.40 & $7.364 \pm 0.005$ & $120 \pm 20$ & $0.037 \pm 0.007$ & $0.00 \pm 0.05$ & $0.28 \pm 0.01$ \\
M 35 & 92.268 & 24.296 & 0.95 & $1.119 \pm 0.002$ & $155 \pm 30$ & $0.228 \pm 0.028$ & $-0.21 \pm 0.10$ & $0.27 \pm 0.01$ \\
NGC 2301 & 102.940 & 0.460 & 0.25 & $1.026 \pm 0.084$ & $180 \pm 40$ & $0.029 \pm 0.001$ & $0.05 \pm 0.05$ & $0.28 \pm 0.01$ \\
M 34 & 40.584 & 42.703 & 0.37 & $1.954 \pm 0.003$ & $210 \pm 40$ & $0.070 \pm 0.000$ & $0.02 \pm 0.06$ & $0.28 \pm 0.01$ \\
M 37 & 88.070 & 32.550 & 0.32 & $0.704 \pm 0.072$ & $450 \pm 100$ & $0.291 \pm 0.050$ & $0.02 \pm 0.05$ & $0.28 \pm 0.01$ \\
M 48 & 123.383 & -5.736 & 0.73 & $1.290 \pm 0.002$ & $500 \pm 150$ & $0.030 \pm 0.010$ & $0.08 \pm 0.03$ & $0.28 \pm 0.01$ \\
Coma Berenices & 185.620 & 25.850 & 7.50 & $11.640 \pm 0.034$ & $600 \pm 50$ & $0.010 \pm 0.010$ & $0.00 \pm 0.08$ & $0.28 \pm 0.01$ \\
Hyades & 66.725 & 15.867 & 13.35 & $21.052 \pm 0.065$ & $750 \pm 50$ & $0.005 \pm 0.005$ & $0.14 \pm 0.02$ & $0.28 \pm 0.01$ \\
Praesepe & 130.090 & 19.690 & 3.00 & $5.371 \pm 0.003$ & $750 \pm 50$ & $0.018 \pm 0.009$ & $0.16 \pm 0.08$ & $0.29 \pm 0.01$ \\
NGC 6811 & 294.310 & 46.390 & 0.30 & $0.873 \pm 0.014$ & $1000 \pm 200$ & $0.062 \pm 0.012$ & $0.03 \pm 0.03$ & $0.28 \pm 0.01$ \\
NGC 752 & 29.520 & 37.810 & 1.45 & $2.330 \pm 0.143$ & $1350 \pm 50$ & $0.040 \pm 0.000$ & $-0.06 \pm 0.03$ & $0.27 \pm 0.01$ \\
NGC 6819 & 295.320 & 40.190 & 0.22 & $0.335 \pm 0.023$ & $2100 \pm 400$ & $0.133 \pm 0.033$ & $0.09 \pm 0.02$ & $0.28 \pm 0.01$ \\
M 67 & 132.848 & 11.837 & 0.31 & $1.133 \pm 0.001$ & $3500 \pm 500$ & $0.039 \pm 0.011$ & $0.03 \pm 0.05$ & $0.28 \pm 0.01$ \\
\hline
\end{tabular}

${ }^{*}$ RA and DEC are the right ascension and declination of the cluster center in degrees, epoch 2000.0; $\Theta$ is the cluster angular radius (degrees); the mean parallax is given in miliarcseconds; ages are given in million year units (Myr); $E(B-V)$ is the color excesses; $[\mathrm{Fe} / \mathrm{H}]$ is the metallicity with respect to the solar value; $Y_{0}$ is the initial helium mass abundance assumed for the evolutionary tracks used to compute physical properties of cluster stars.

Rotational periods of field stars were computed by several research groups using observations from the CoRoT satellite (Affer et al. 2012), the HATNet survey for transiting extrasolar planets (Hartman et al. 2011), and the Kepler satellite project (Nielsen et al. 2013; Reinhold et al. 2013; McQuillan, Mazeh \& Aigrain 2014).

To homogenize the data set, the Vizier $^{1}$ facility was used to collect the spectral type, visual magnitude and classification of each star as reported by SIMBAD ${ }^{2}$, their $G, B P$ and $R P$ band magnitudes and parallax from Gaia DR2 ${ }^{3}$, and their $J$, $H$ and $K$ magnitudes from the Two Micron All Sky Survey $^{4}$ (herein $2 M A S S$ ).

\footnotetext{
${ }^{1}$ This research has made use of the VizieR catalogue access tool, CDS, Strasbourg, France (Ochsenbein, Bauer \& Marcout 2000) and the cross-match service provided by CDS.

${ }^{2}$ This research has made use of the SIMBAD database, operated at CDS, Strasbourg, France (Wenger et al. 2000).

${ }^{3}$ This work has made use of data from the European Space Agency (ESA) mission Gaia, processed by the Gaia Data Processing and Analysis Consortium. Funding for the DPAC has been provided by national institutions, in particular the institutions participating in the Gaia Multilateral Agreement.

${ }^{4}$ This publication makes use of data products from the Two Micron All Sky Survey, which is a joint project of the
}

This information is collected in a number of tables containing stellar coordinates (degrees, epoch 2000; precise within 1 arcsec), spectral types ('*?' when unknown), visual magnitude as reported in SIMBAD (as a reference), Johnson-Cousins visual magnitudes calculated from the Gaia DR2 $G, B P$ and $R P$ band magnitudes and the transformation laws found by Evans et al. (2018), J, $H$ and $K$ $2 M A S S$ magnitudes, rotational periods (days) and Gaia DR2 parallaxes (miliarcsec). When there is no information for any of these quantities, the corresponding field value is 999.9 .

These tables include all the stars reported in each rotational data source. Rotational periods in the Kepler field and the Pleiades, M 34, Hyades and Praesepe open clusters were searched for by more than one research group. Additional tables excluding duplicate stars were produced for these clusters and the field. Mean periods are reported for stars with multiple measurements, with the period error being

University of Massachusetts and the Infrared Processing and Analysis Center/California Institute of Technology, funded by the National Aeronautics and Space Administration and the National Science Foundation. 
equal to half the difference between the shortest and longest period.

Several filters were applied to select stars with good photometric and astrometric data quality and no possible caveats regarding contamination from other motives of variability besides single star rotation. As explained in the following subsection, distinctive flags are added when these filters are activated.

\subsection{Data Filters}

A flag following the visual magnitude derived from the Gaia data is set to 'XX' under column GP, when it could not be calculated because at least one of the Gaia DR2 G, BP and RP magnitudes and their errors is missing, or the photometric flux over error in any of these bands is smaller than 5. Otherwise, the Gaia flag $G P$ is set to '-'.

A flag following $2 M A S S$ magnitudes is set to 'XX' under column 2P if either one of the $J, H$ and $K$ magnitudes and their errors is absent, the photometric flux over error in any band is smaller than 5 , the $2 M A S S$ quality flag is not set to 'AAA' and the star may be contaminated by an extended source (their Xflg is larger than zero). Otherwise, the $2 M A S S$ flag $2 \mathrm{P}$ attached to the $J, H$ and $K$ magnitudes is set to '-'.

Databases were used to identify possible non main sequence stars, object types that may indicate that the angular momentum evolution of a single star may have been affected by an external agent (such as stellar companions and planetary systems) or light curve modulations produced by other sources of variability besides single star rotation. Namely, the following types (in parenthesis, condensed name for object classification recommended by SIMBAD):

- stars with known exoplanets $\left(\mathrm{Pl}^{*}\right)$ as of 02-072019, according to the NASA Exoplanet Science Institute $^{5}$,

- double or multiple stars $\left(* *, *^{*}\right)$ and spectroscopic binaries $\left(\mathrm{SB}^{*}\right)$, according to the Ninth Catalogue of Spectroscopic Binary Orbits (Pourbaix et al. 2004), SIMBAD, Washington Double Star Catalog 6 and other sources,

\footnotetext{
${ }^{5}$ This research has made use of the NASA Exoplanet Archive, which is operated by the California Institute of Technology, under contract with the National Aeronautics and Space Administration under the Exoplanet Exploration Program

${ }^{6}$ This research has made use of the Washington Double Star Catalog maintained at the U.S. Naval Observatory.
}

- Algol, $\beta$-Lyrae, W-Uma, RS CVn and X-ray and eclipsing binaries $\left(\mathrm{Al}^{*}, \mathrm{bL}^{*}, \mathrm{WU}^{*}, \mathrm{RS}^{*}, \mathrm{XB}^{*}\right.$, $\mathrm{EB}^{*}, \mathrm{~EB}^{*}$ WUma, EB*betLyr, , according to SIM$\mathrm{BAD}$ and other sources,

- ellipsoidal, symbiotic, cataclysmic, pulsating, $\delta$-Scuti, $\gamma$-Doradus, RR-Lyrae, classical Cepheid, Cepheid, SX Phe and Mira and long period variables $\left(\mathrm{El}^{*}, \mathrm{Sy}^{*}, \mathrm{CV}^{*}, \mathrm{Pu}^{*}, \mathrm{dS}^{*}, \mathrm{gD}^{*}, \mathrm{RR}^{*}, \mathrm{cC}^{*}\right.$, $\left.\mathrm{Ce}^{*}, \mathrm{SX}^{*}, \mathrm{LPV}^{*}\right)$, according to SIMBAD, Holl et al. (2018) and other sources,

- brown dwarfs $\left(\mathrm{BD}^{*}\right)$ and T-Tauri stars $\left(\mathrm{TT}^{*}\right)$, according to SIMBAD and

- not in main sequence, according to SIMBAD and other sources (No-MS*; this abbreviation is not used by SIMBAD).

When present, these name tags are reported under column label Comment. This Comment may also include the following name tags

- XPlx if there is no Gaia DR2 parallax measurement, the parallax error is larger than $20 \%$, the astrometric excess noise (epsi) is larger than 2, the used visibility periods (Nper) are less than 8 and duplicate sources (Dup) may be present,

- XPer when no rotational period is measured or the rotational period is reported as questionable or the period-to-period-error ratio is smaller than 5. When there is more than one data source as in the Hyades, M 34, Pleiades and Praesepe clusters, as well as in the Kepler field stars - the period is assumed to be equal to the mean value and the period error is taken as half the difference between the shortest and longest period. Period uncertainty was significant in stars from the Kepler field and the M 34 and Pleiades open clusters (4925, 18 and 36 rejections), implying that formal errors in the mathematical analysis may underestimate other basic sources of uncertainty in the determination of rotational periods. Some sources of uncertainty may be associated to the wavering nature of stellar activity. Unfortunately, errors in the reported rotational period are not given in a large number of papers, so that an indefinite number of stars with less than adequate period measurements could not identified.

The final comment is set to '-' when the star has not been associated to any of the preceding types and the stellar parallax and period have been adequately measured. There are electronic versions of tables 
TABLE 2

COMA BERENICES. DATA

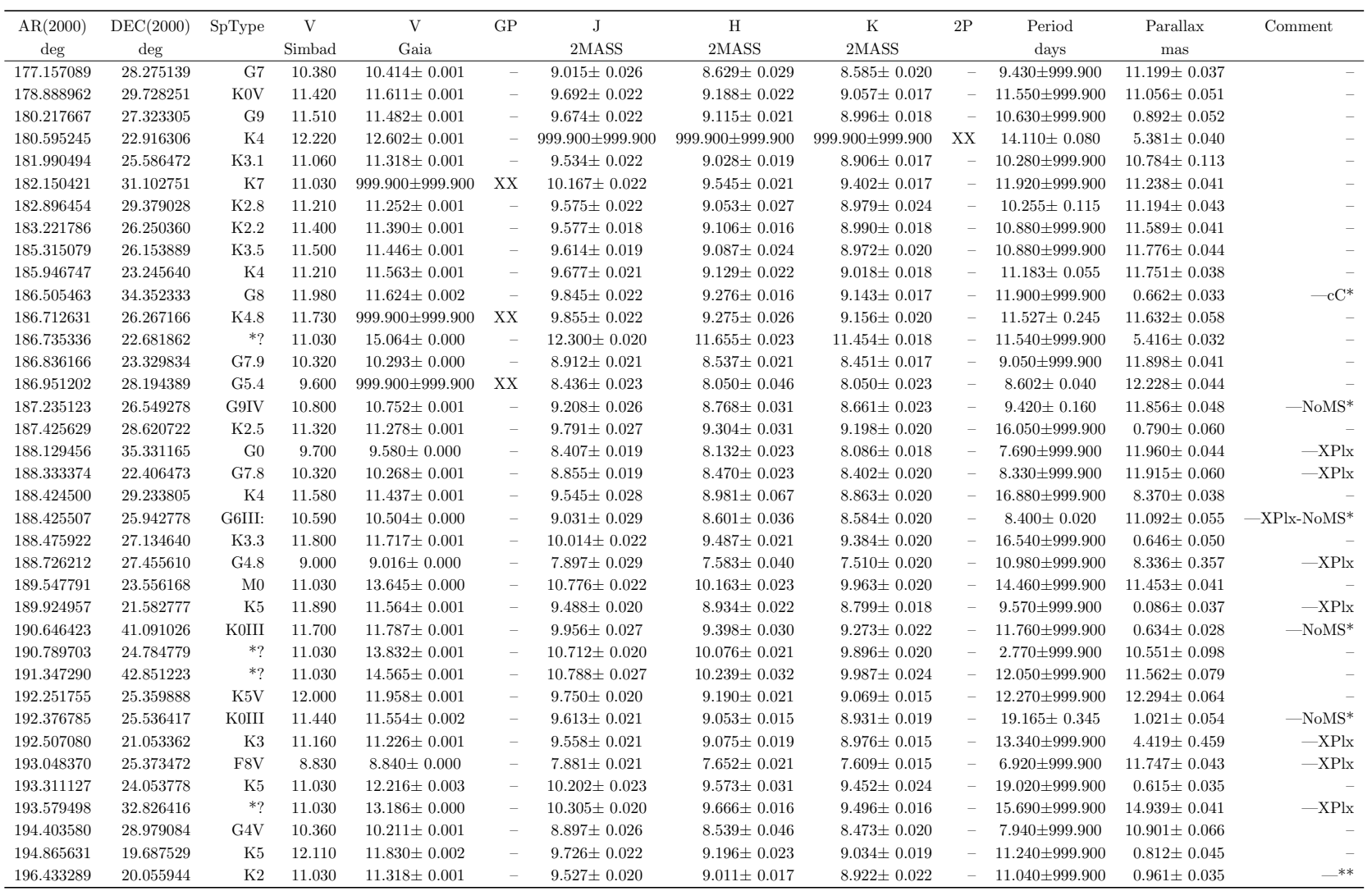

for primary data from field and open cluster stars (http://vizier.u-strasbg.fr/viz-bin/VizieR?-source= J/other/RMxAA/56.1, ftp://cdsarc.u-strasbg.fr/ $\mathrm{pub} /$ cats/J/other/RMxAA/56.1). As an example, see Table 2 .

The number of stars with measured rotational periods in each cluster and field is given under Column Per in Table 3. The number of stars that were selected after passing all data filters is under Column $O K$ in the same table. Notice that there is a large fraction of rejected stars in most open clusters. There are many reasons for this to be so, but the most frequent is insufficiently accurate parallax (94, 60 and $47 \%$ in h Persei, M 37 and NGC 6819), generally in clusters that are farther away. Stellar parallax is also poor in a large fraction of stars associated to the two open clusters that are closer to us, Hyades and Coma Berenices (43 and 21\%). The next two main reasons for rejection are poor or non-existent $2 M A S S$ photometry and association to a multiple system, with very high levels of incidence in h Persei, IC 4665 and NGC 6819. Parallax was too inaccurate in $59 \%$ of the HATNet field stars, and $42 \%$ of the
CoRoT field stars are not in the main sequence. Fortunately, all these problems affected a much smaller fraction of Kepler field stars.

In total, the rotational period has been measured for 3769 members of the 17 clusters listed in Table 1 , and for 45235 field stars observed by the CoRoT, HATNet and Kepler projects. Out of these, the data filters approved 1876 cluster and 32641 field stars.

\subsection{Extinction Correction}

Gaia DR2 provides an estimate of line-of-sight extinction, $A_{G}$, and reddening, $E(B P-R P)$ for each star. After a careful analysis of the Gaia DR2 database, Andrae et al. (2018) concluded that these line-of-sight extinction estimates are not accurate at the single star level and, concurrently, that there are no clearly defined transformations between $A_{G}$ and $A_{V}$, and $E(B P-R P)$ and $E(B-V)$. Consequently, absolute magnitudes of open cluster stars were determined assuming that the color excess is the same for all stars and is as given in Table 1. This is a particularly weak assumption in young clusters, where dust distribution can be very inhomogeneous at the 
TABLE 3

STARS IN EACH CLUSTER AND FIELD*

\begin{tabular}{|c|c|c|c|c|c|c|c|c|c|}
\hline Cluster & Per & $O K$ & $V I R$ & $I R$ & Cluster & Per & $O K$ & $V I R$ & $I R$ \\
\hline h Persei ${ }^{a}$ & 586 & 20 & 19 & 19 & IC $4665^{b}$ & 19 & 6 & 0 & 5 \\
\hline NGC $2547^{c}$ & 176 & 152 & 6 & 110 & Blanco $1^{d}$ & 33 & 23 & 19 & 20 \\
\hline Pleiades-C ${ }^{e}$ & 132 & 70 & - & - & Pleiades- $\mathrm{H}^{f}$ & 516 & 368 & - & - \\
\hline Pleiades-R $\mathrm{R}^{g}$ & 759 & 611 & - & - & Pleiades-All & 997 & 741 & 64 & 320 \\
\hline M $35^{h}$ & 441 & 297 & 82 & 282 & NGC $2301^{i}$ & 133 & 83 & 83 & 82 \\
\hline M $34-\mathrm{I}^{j}$ & 83 & 60 & - & - & M 34-J ${ }^{k}$ & 55 & 34 & - & - \\
\hline M $34-\mathrm{M}^{l}$ & 120 & 22 & - & - & M 34-All & 243 & 102 & 89 & 102 \\
\hline $\mathrm{M} 37^{m}$ & 657 & 125 & 31 & 119 & M $48^{n}$ & 54 & 51 & 51 & 51 \\
\hline Coma $\mathrm{Ber}^{\circ}$ & 37 & 20 & 6 & 8 & Hyades-De $^{p}$ & 62 & 30 & - & - \\
\hline Hyades-Do ${ }^{q}$ & 48 & 14 & - & - & Hyades- $\mathrm{R}^{r}$ & 22 & 6 & - & - \\
\hline Hyades-All & 123 & 58 & 7 & 19 & Praesepe-A ${ }^{s}$ & 40 & 20 & - & - \\
\hline Praesepe- $\mathrm{D}^{t}$ & 52 & 0 & - & - & Praesepe-S ${ }^{u}$ & 54 & 36 & - & - \\
\hline Praesepe-All & 137 & 114 & 28 & 90 & NGC $6811^{v}$ & 71 & 53 & 52 & 52 \\
\hline NGC $752^{w}$ & 12 & 8 & 8 & 8 & NGC $6819^{x}$ & 30 & 7 & 6 & 7 \\
\hline M $67^{y}$ & 20 & 16 & 16 & 16 & & & & & \\
\hline Field & Per & $O K$ & $V I R$ & $I R$ & Field & Per & $O K$ & $V I R$ & $I R$ \\
\hline CoRoT $^{a}$ & 1978 & 781 & 694 & 761 & HATNet $^{b}$ & 2018 & 704 & 176 & 322 \\
\hline Kepler-M ${ }^{c}$ & 34186 & 28507 & - & - & Kepler- $\mathrm{N}^{d}$ & 12151 & 9841 & - & - \\
\hline Kepler- $\mathrm{R}^{e}$ & 24124 & 20027 & - & - & Kepler-All & 41239 & 31156 & 27494 & 29032 \\
\hline
\end{tabular}

*Per: stars with measured rotational periods. OK: stars that passed all data filters. VIR: stars where the visual and infrared magnitudes were matched by a stellar model. IR: stars where only the infrared magnitudes were reproduced by a stellar model. Cluster period data: (a) Moraux et al. 2013; (b) Scholz, Eislöffel \& Mundt 2009; (c) Irwin et al. 2008; (d) Cargile et al. 2014; (e) Covey et al. 2016; (f) Hartman et al. 2010; (g) Rebull et al. 2016; (h) Meibom, Mathieu \& Stassun 2009; (i) Sukhbold \& Howell 2009; (j) Irwin et al. 2006); (k) James et al. 2010; (l) Meibom et al. 2011; (m) Messina et al. 2008, Hartman et al. 2009 \& Nuñez et al. 2015; (n) Barnes et al. 2016; (o) Collier Cameron et al. 2009; (p) Delorme et al. 2011; (q) Douglas et al. 2016; (r) Radick et al. 1987 \& 1995; (s) Agüeros et al. 2011; (t) Delorme et al. 2011; (u) Scholz et al. (2011); (v) Meibom et al. 2011; (w) Agüeros et al. 2018; (x) Meibom et al. 2015; (y) Barnes et al. (2016). Field period data: (a) Affer et al. 2012; (b) Hartman et al. 2011; (c) McQuillan, Mazeh \& Aigrain 2014; (d) Nielsen et al. 2013; (e) Reinhold et al. 2013.

intra-cluster and individual level. For field stars, the extinction model is a smooth vertically-exponential dust disk, and the extinction coefficient at galactic latitude $b$ and distance $d$ is

$$
C(b)=C(0)[1-H \exp (-d \sin b / h) / \sin b)],
$$

where $C(0)$ is the mid-plane extinction coefficient and $h$ is the model scale-height, which is taken as equal to $0.14 \mathrm{kpc}$ (Koppen \& Vergely 1998; Marshal et al. 2006; Brown et al. 2011). The assumed midplane extinction coefficients are $C_{V}=1.0, C_{J}=0.35$, $C_{H}=0.25$ and $C_{K}=0.15$ magnitudes per kiloparsec (Indebetouw et al. 2005). Implicitly, line of sight variations are supposed to be a minor correction.

\subsection{Stellar Parameters}

Stellar parameters were inferred matching (within error bars) the observed absolute magnitudes in the Johnson-Cousins $V$ band, and the Bessel \& Brett $J, H$ and $K$ bands, with those deduced from the latest Yale-Potsdam stellar isochrones (Spada et al. 2013; Spada et al. 2017, herein YaPSI) and the semiempirical color-temperature calibration of Worthey \& Lee (2011). The $2 M A S S$ magnitudes were converted to the Bessel \& Brett system using formulae derived by Carpenter (2001). The parameter space of YAPSI models is dense enough to carry out detailed interpolations over a broad spectrum of possibilities: from the birth line to the onset of helium 
ignition in the core, 0.15 to $5.0 M_{\odot},[\mathrm{Fe} / \mathrm{H}]=-1.5$ to 0.3 and $Y_{0}=0.25$ to 0.37 . Notice that these are iron and helium abundances at the birth line, not the larger values they have at a posterior time. Besides the usual parameters (mass, gravity, luminosity, temperature, etc.), the YaPSI archives also include the depth of the convective envelope, the convective overtime timescale and the moments of inertia of the entire star and its convective envelope. These quantities are essential in the understanding of the evolution of angular momentum, magnetic activity and mass loss.

The physical parameters of open cluster stars are assumed to be equal to the mean values of models having 27 different age, $[\mathrm{Fe} / \mathrm{H}]$ and Y0 combinations, with each of these parameters being equal to the minimum, mean and maximum values given in Table 1. For instance, the YaPSI models used for the Pleiades are 27 combinations of three age estimates (100, 120 and $140 \mathrm{Myr}$ ), three initial iron abundances $(-0.05,0.00$ and 0.05$)$ and three initial helium abundances $(0.27,0.28$ and 0.29$)$. No attempt is made to estimate and use the initial abundances of iron and helium, but these are probably close to the minimum values. In Table 3, the number of stars with a model accounting for their visual and infrared magnitudes is under Column VIR, and in Column IR when only the infrared magnitudes were matched. To exclude multiple observations of individual stars, data files from the Pleiades, M 34, Hyades and Praesepe clusters were merged into one (name-All).

The visual and infrared magnitudes of a substantial fraction of stars in the Hyades $(65 \%)$, Pleiades (57\%) and NGC 2547 (29\%) open clusters could not be reproduced with any of the corresponding YaPSI models, suggesting that there may be larger than explored differences in the chemical makeup of stars that are thought to be part of these open clusters and/or that extinction corrections were inadequate in a large number of them. In this respect, model matching including visual magnitudes was substantially more ineffective in IC 4665, NGC 2547, Pleiades, M 37, M 35, Praesepe and Hyades, which may imply that uniform extinction is an inadequate assumption for these relatively young associations. Notice that this does not hold in every young cluster (for instance, h Persei, M 48, NGC 2301 and Blanco 1) and certainly not in those that are older than 1000 Myr. Fluctuations in the predicted values of all physical parameters were less than $10 \%$ in nearly all open cluster stars. The most notable exception is NGC 2301, where all predicted stellar parameters fluctuate more than $30 \%$ in every star.

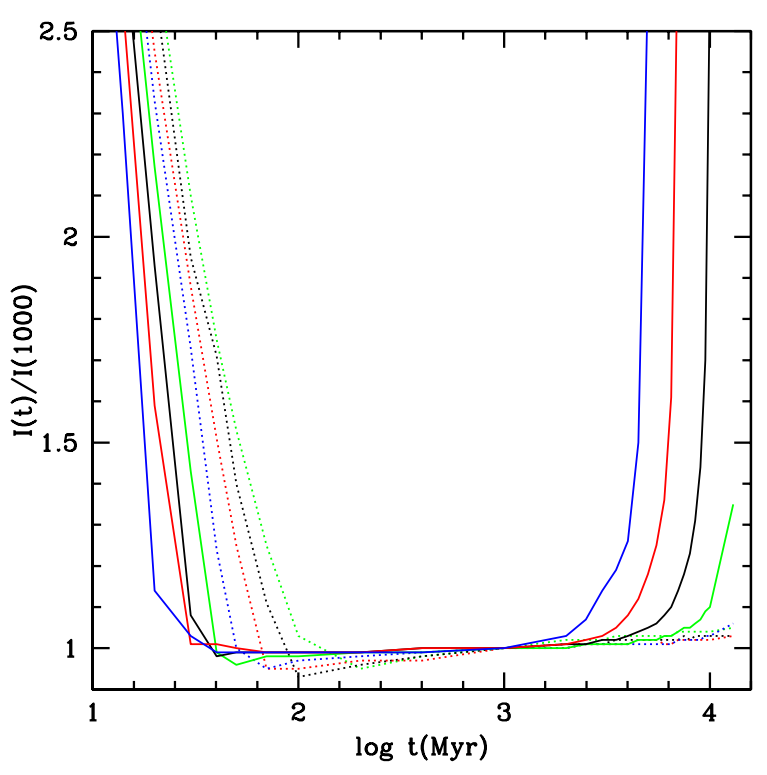

Fig. 1. Ratio of the moment of inertia at time $t, I(t)$, with respect to the moment of inertia at $t=1000 \mathrm{Myr}$. Dotted lines are for $M / M_{\odot}=0.5$ (green), 0.6 (black), 0.7 (red) and 0.8 (blue). Continuous lines are for $M / M_{\odot}$ $=0.9$ (green), 1.0 (black), 1.1 (red) and 1.2 (blue). The color figure can be viewed online.

Model results were equally discouraging in 1 out of 4 stars from the Hyades and 3 out of 7 in NGC 6819. Unfortunately, being more massive than $1.35 M_{\odot}$, the few remaining stars from the relatively and conveniently old NGC 6819 open cluster are probably not subject to rotational braking. To use a sufficiently large amount of data, the impending analysis will include cluster stars where the visual magnitude could not be matched.

The physical parameters of field stars were extracted after inspecting a set of models with an initial close to solar-like metallicity and helium abundance $\left([\mathrm{Fe} / \mathrm{H}]=0.0\right.$ and $\left.Y_{0}=0.28\right)$ and 37 possible ages, from 1 to $13000 \mathrm{Myr}$. The observed period was used as a roughly confined mass dependent age restriction. This is imperative, since there are important changes in the moment of inertia at the beginning (first $\approx 100 \mathrm{Myr}$ ) and during the second half of the main sequence, as can be appreciated in Figure 1.

The visual and infrared magnitudes of a very small fraction of HATNet field stars with high quality data and no other possible sources of variability could be reproduced with a YaPSI model, and nearly half of these models were theoretically inaccurate at the $30 \%$ level or more. A stellar model matching visual and infrared magnitudes could be found for the majority of CoRoT field stars that survived the 


\section{TABLE 4}

COMA BERENICES. PARAMETERS*

\begin{tabular}{ccccccccccc}
\hline $\mathrm{AR}(2000)$ & $\mathrm{DEC}(2000)$ & $M M_{\odot}$ & $T$ & $L / L_{\odot}$ & $R / R_{\odot}$ & $\log g$ & \multicolumn{1}{c}{$I_{e} / I_{t}$} & \multicolumn{2}{c}{ Period } & $J_{47}$ \\
\hline 178.888962 & 29.728251 & $0.79 \pm 0.01$ & $4780 \pm 47$ & $0.240 \pm 0.006$ & $0.71 \pm 0.00$ & $4.62 \pm 0.00$ & $0.26 \pm 0.01$ & $11.55 \pm 999.90$ & $28.821 \pm 0.992$ & 7 \\
183.221786 & 26.250360 & $0.77 \pm 0.00$ & $4842 \pm 11$ & $0.244 \pm 0.001$ & $0.70 \pm 0.00$ & $4.63 \pm 0.00$ & $0.24 \pm 0.00$ & $10.88 \pm 999.90$ & $28.544 \pm 0.538$ & 2 \\
185.315079 & 26.153889 & $0.78 \pm 0.01$ & $4734 \pm 60$ & $0.226 \pm 0.007$ & $0.71 \pm 0.00$ & $4.63 \pm 0.00$ & $0.26 \pm 0.01$ & $10.88 \pm 999.90$ & $29.915 \pm 1.404$ & 6 \\
186.735336 & 22.681862 & $0.61 \pm 0.01$ & $4047 \pm 47$ & $0.079 \pm 0.002$ & $0.57 \pm 0.01$ & $4.70 \pm 0.00$ & $0.36 \pm 0.01$ & $11.54 \pm 999.90$ & $17.351 \pm 0.943$ & 19 \\
186.836166 & 23.329834 & $0.90 \pm 0.00$ & $5445 \pm 25$ & $0.514 \pm 0.007$ & $0.81 \pm 0.00$ & $4.58 \pm 0.00$ & $0.17 \pm 0.01$ & $9.05 \pm 999.90$ & $44.585 \pm 0.406$ & 2 \\
189.547791 & 23.556168 & $0.58 \pm 0.01$ & $3963 \pm 41$ & $0.065 \pm 0.001$ & $0.54 \pm 0.01$ & $4.73 \pm 0.00$ & $0.39 \pm 0.01$ & $14.46 \pm 999.90$ & $12.206 \pm 0.621$ & 3 \\
190.789703 & 24.784779 & $0.63 \pm 0.00$ & $4095 \pm 14$ & $0.089 \pm 0.001$ & $0.59 \pm 0.00$ & $4.69 \pm 0.00$ & $0.35 \pm 0.00$ & $2.77 \pm 999.90$ & $78.544 \pm 1.405$ & 4 \\
194.403580 & 28.979084 & $0.95 \pm 0.01$ & $5641 \pm 84$ & $0.660 \pm 0.029$ & $0.85 \pm 0.00$ & $4.55 \pm 0.00$ & $0.14 \pm 0.01$ & $7.94 \pm 999.90$ & $55.409 \pm 2.500$ & 6 \\
\hline
\end{tabular}

${ }^{*} I_{e} / I_{t}$ is the envelope-to-total moment of inertia ratio. $J_{47}$ is the solid body angular momentum in units of $10^{47} \mathrm{gr} \mathrm{cm}^{2} / \mathrm{s}$. $\mathrm{NM}$ is the number of models that matched the infrared and visual magnitudes.

data filters, but more than half of them were also inexact to a very high level. To exclude multiple observations, Kepler field data files were merged into Kepler-All. At least one stellar model was able to account for the visual and/or infrared magnitudes of $\approx 90 \%$ of main sequence Kepler field stars. The predicted mass, temperature, radius and gravity (its logarithm) of the vast majority of these stars (at least 8 out of 10) is theoretically accurate at the $90 \%$ level or better, and less than $66 \%$ certain in roughly 1 out of 40 stars. The moment of inertia is precise at the $90 \%$ level in 8 out of 10 stars, but very uncertain in 1 out of 10 . Results are slightly worse for the stellar luminosity. Since many more Kepler field stars were observed with a higher quality and success rate, CoRoT and HATNet field stars will no longer be considered. Being more reliable, the ensuing analysis will only deal with Kepler field stars for which the visual and infrared magnitudes are reproduced.

At least one model matched the infrared magnitudes of 1311 and 30115 open cluster and field stars, and the visual and infrared magnitudes of 566 and 28364 of these stars. Electronic versions of tables with physical parameters of stars in each cluster and field are available upon request (http://vizier.u-strasbg.fr/viz-bin/VizieR?-source= J/other/RMxAA/56.1, ftp://cdsarc.u-strasbg.fr/ $\mathrm{pub} /$ cats/J/other/RMxAA/56.1). As an example, see Table 4.

\section{ANALYSIS AND DISCUSSION}

\subsection{Rotational Period as a Function of Mass and Time. First Round.}

With their data, McQuillan, Mazeh \& Aigrain (2014) produced a figure for the logarithm of the rotational period as a function of mass for Kepler field stars (their Figure 1). Almost all periods are within rather well defined lower and upper mass dependent limits. Referring to empirical gyrochronology models, they mention that the upper envelope period distribution is broadly consistent with an age of $4500 \mathrm{Myr}$. The rotational period lower boundary was not discussed and, consequently, no age estimate was provided for this border.

McQuillan, Mazeh \& Aigrain (2014) also point out that their figure exhibits a bimodal period distribution for stellar masses less than $\approx 0.6 M_{\odot}$. The existence of large dispersions and bimodality in the rotation rates of young open cluster $G$ and $K$ main sequence stars has been noticed for some time (Soderblom, Jones \& Walker, 1983). Tracing fast and moderate-to-slow rotation sequences in several young clusters, Meibom et al. (2011a) found that this distribution tends to vanish in older clusters, with more massive stars being more likely to converge on a single rotational sequence. The bimodal period distribution of late $\mathrm{K}$ and $\mathrm{M}$ dwarf field stars is in line with this observation.

The rotational period as a function of mass for the Kepler field stars that passed all data filters and had their visual and infrared colors reproduced by a YaPSI model (as described above), is shown in the upper panel of Figure 2. The Sun, $\alpha$ Cen A and B (4850 \pm 500 Myr; Bazot et al. 2007; Dewarf, Datin \& Guinan 2010) and 16 Cygnus A and B (6800 \pm 400 Myr; Davies et al. 2015) are included in this figure. McQuillan, Mazeh \& Aigrain (2014) introduced a limit of $T_{\text {eff }}<6500 \mathrm{~K}$ in their analysis, since they were only interested in stars with convective envelopes. When this limit is not introduced, an unanticipated result is a large number of slow rotators that are more massive than $\approx 1.3 M_{\odot}$. A smaller number of such stars are also found in the Reinhold et al. (2013) analysis of the Kepler database. If on the main sequence, these stars are predicted to be rotating more rapidly, since they have a very shallow convective envelope (if at all) and therefore no enhanced magnetic field to lessen their angular momentum. On the other hand, longer periods and lower temperatures are expected if they are turning 

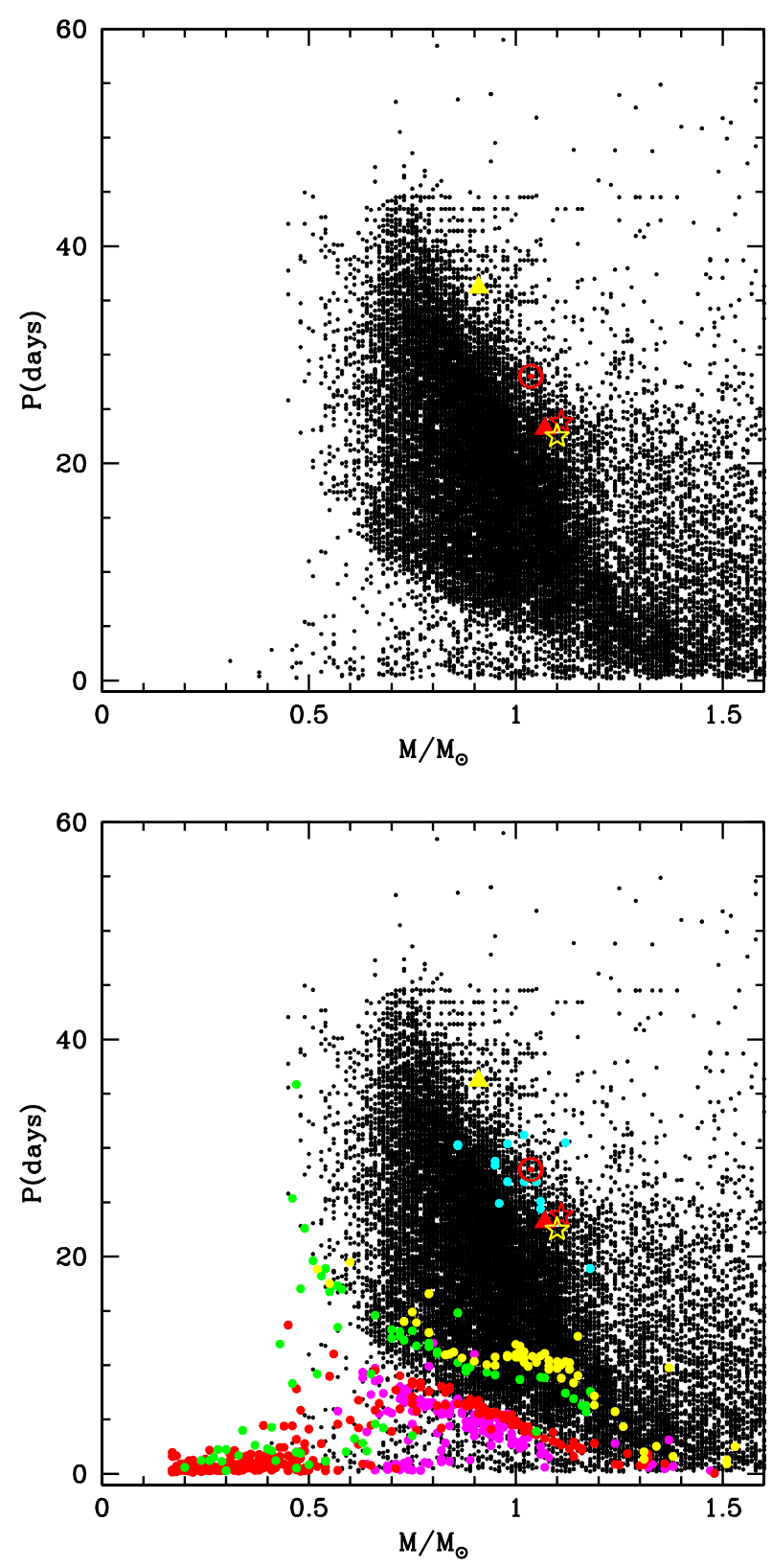

Fig. 2. Rotational period (P, days) as a function of mass (solar units). Upper and lower panel: Kepler field stars (black). Lower panel: stars from the Pleiades (red, 120 Myr), M 35 (magenta, 155 Myr), Praesepe (green, 750 Myr), NGC 6811 (yellow, 1000 Myr), NGC 752 (yellow, $1350 \mathrm{Myr}$ ) and M 67 (cyan, $3500 \mathrm{Myr}$ ) open clusters. Only open cluster stars with errors less than $10 \%$ in their observational parameters are shown in this figure. Open stars and filled triangles are for $\alpha$ Cen A and B (yellow) and 16 Cygnus A and B (red). The data for these stars are in Table 6 . The color figure can be viewed online. away from main sequence. This combination is found in a number of cases. As can be seen in Figure 2, very few stars less massive than $\approx 0.5 M_{\odot}$ were left after the data filters were applied and this may be the reason why the bimodal period distribution of low mass field stars is no longer apparent. Besides these two questions, there are no other overall differences between this figure and Figure 1 in McQuillan, Mazeh \& Aigrain (2014).

Most of the lower boundary of the cone defined by the Kepler field stars draws the rotational period of stars once they have stabilized in the main sequence, sometime after reaching a steady rotational shear and moment of inertia. An age estimate of the lower boundary for the period distribution can be established plotting the period as a function of mass in stars from young open clusters with known age. The lower panel of Figure 2 also contains the rotational periods of stars from several open clusters. To avoid cluttering, only open cluster stars with errors less than $10 \%$ in their observational parameters are shown in this figure. The same conclusions are obtained when all the stars used in the numerical analysis (errors less than 20\%) are included in this plot. It is quite remarkable that most of the lower boundary coincides with the position occupied by slowly rotating Pleiades stars, setting the location of a zero age rotational main sequence for late type stars at approximately 120 Myr after birth. The location of slow rotators in the M 35 cluster runs in parallel but with slightly shorter periods, suggesting that this cluster is not $155 \mathrm{Myr}$ old, but younger than the Pleiades.

There is a single, well defined, mass dependent period distribution in the Pleiades, M 35 and Praesepe open clusters, as long as the stellar mass is larger than $\approx 0.8 M_{\odot}$. Early convergence to a single period distribution in the high mass range is supported by the fact that no fast $0.7-1.2 M_{\odot}$ rotators were found in M 37 (450 Myr) and M 48 (500 Myr). On the other hand, M 34 is a $210 \mathrm{Myr}$ old open cluster supporting the notion that convergence to a single distribution in this mass range may take more than $120 \mathrm{Myr}$; the rotational periods of most Pleiades and M 34 stars more massive than $0.8 M_{\odot}$ are comparable, but quite a few stars in M 34 are fast rotators with significantly lower periods than Pleiades and M 35 stars with a similar mass. Unfortunately, in NGC 2301 (180 Myr) there are no rotational periods for stars more massive than $\approx 0.8 M_{\odot}$. In conclusion, high quality data indicate that after $\approx 120 \mathrm{Myr}$, most $\approx 0.8-1.2$ main sequence stars converge to the rotational sequence defined by 
the Pleiades. This is earlier than previously thought (around $600 \mathrm{Myr}$ ). One of the possible reasons for this discrepancy is that past estimates used a larger mass bin (at least $0.2 M_{\odot}$ in Gallet \& Bouvier 2013 and 2015) and combined data from clusters with different ages (Johnstone et al. 2015a). Notice that the dispersion - and the impression that there is no convergence - will increase when these operations are carried out. Finally, there are signs of a bimodal period distribution in Pleiades, M 35 and Praesepe $(750 \mathrm{Myr})$ stars less massive than $\approx 0.8 M_{\odot}$, but no indication of such a thing in older clusters. Notice that there are relatively few fast rotators in Praesepe and, at least down to $\approx 0.5 M_{\odot}$, apparently none in clusters older than $750 \mathrm{Myr}$, such as NGC 6811 (1000 Myr) and NGC 752 (1350 Myr). Thus, nearly all $0.5-0.8 M_{\odot}$ stars seem to merge into a single mass dependent rotational evolutionary sequence sometime after $750 \mathrm{Myr}$.

The relative position of the rotational period distributions of stars from the Pleiades, M 35, Praesepe, NGC 6811 (1000 Myr), NGC 752 (1350 Myr) and M 67 (3500 Myr) open clusters leaves no doubt that there is an evolutionary sequence. Stars from the oldest open cluster depicted in this figure, M 67 , do not draw a similarly clear continuous line as the Pleiades stars, and the upper boundary (long periods) drawn by Kepler field stars is not as well defined. An interesting point is that $\alpha$ Cen $\mathrm{A}$ and $\mathrm{B}$, which are $\approx 1000$ older than M 67 , are close to the site occupied by stars from this open cluster. This indicates that once they merge into a single rotational sequence, angular momentum evolution of single solar type stars (mass between 0.9 and $1.1 M_{\odot}$ ) is weakly dependent on binarity when the orbital period is sufficiently long (79 years in $\alpha$ Cen AB). Additionally, it implies that rotational braking of solar type stars is increasingly inefficient as they grow old. The second conclusion is strongly supported by the fact that there is hardly any difference between the rotational period of these stars and 16 Cygnus $\mathrm{A}$ and $\mathrm{B}$, which are $\approx 3000 \mathrm{Myr}$ older than the M 67 cluster. It is worth noting that the long-term rotational evolution of the Sun as a single star does not seem to have been affected in a major way by our planetary system. It remains to be seen which conditions are necessary for this to happen.

\subsection{Rigid Body Angular Momentum as a Function of Mass and Time}

Since angular momentum and rotational energy are the physical quantities that change during stellar evolution, we can not expect a clearly defined signa- ture for the mass and time dependence of the mean rotational period at the stellar surface. A comprehensive and definite knowledge of these quantities - angular momentum and energy - is still beyond reach, since little is known on the radial dependence of the angular velocity as a function of stellar mass and age. Assumptions have to be made, and these are greatly influenced by what we know about the Sun, which is well advanced into its rotational history (as evinced in Figure 2).

Helioseisimology has shown that the radiative core of the Sun rotates rigidly at a rate of $431 \mathrm{nHz}$ (period close to 27 days) at least down to $\approx 0.15 R_{\odot}$ (Kozennik \& Eff-Darwich, 2012; Eff-Darwich \& Korzennik, 2013). Taking a radial average, the convective envelope rotates at a similar rate (or slightly higher according to Tomczyk, Schou \& Thompson 1995). Since the outer layers are slowed down by magnetic braking, this signifies that angular momentum transport from the radiative core to the convective envelope has been equally efficient for some time.

But not much is known on the internal rotation of other stars, and Lund, Miesch \& ChristensenDalsgard (2014) argued that asteroseismological measurements of Sun-like stars cannot result in clean-cut inferences on the radial profile of the rotational period. Even so, Nielsen et al. (2017) concluded that the interior and envelope rotation rates cannot differ by more than $\approx 30 \%$ in 5 solar-like stars (insofar as mass and age are concerned), and Collier Cameron et al. (2009) claim that F, G and $\mathrm{K}$ main sequence stars rotate as rigid bodies after $\approx 600$ Myr. Fully convective main and pre-main sequence stars are expected to rotate as rigid bodies, and Charbonnel et al. (2013) showed that the rotational frequency of a solar mass ZAMS star peaks at $\approx 0.25 R_{\odot}$ and is approximately constant beyond $\approx 0.5 R_{\odot}$, if angular momentum is redistributed by internal gravity waves.

Modeling the rate at which angular momentum is lost at the stellar surface, and examining the constraints on internal angular momentum transport which can be inferred from the $0.2 M_{\odot}$ mass bin period distributions of several clusters, Denissenkov et al. (2010) concluded that $0.4 \leq M / M_{\odot} \leq 1.2$ stars with an initial rotation period of less than $\approx 2$ days, will rotate as rigid bodies during most of their main sequence evolutionary stage. But if the initial period is between 2 and 4 days, the core-envelope coupling timescale will be $50 \pm 25 \mathrm{Myr}$ when $M=1.0 \pm 0.1 M_{\odot}$, or $175 \pm 25 \mathrm{Myr}$ when $M=0.8 \pm 0.1 M_{\odot}$. More extended periods lead to longer coupling times. Notice 
that by the time they reach the zero age rotational main sequence, $\approx 120 \mathrm{Myr}$ after birth, the rotational period is close to 10 days when $M / M_{\odot} \simeq 0.7$ and less than 5 days if $M / M_{\odot}>1$ (see Figure 2). Obviously, the initial rotational periods had to be smaller.

From the preceding paragraphs, it seems that a rigid rotation model may be not too far-off from reality. It may even be an almost precise description of stars that are older than a few hundred Myr, but not too close to the end of their main sequence lifetime. Needless to say, rigid body rotation is also the simplest way to carry out an inspection of angular momentum evolution. The rest of this paper will discuss this subject, under the hypothesis that late-type main sequence stars rotate as rigid bodies. Arguing that there seems to be moderate differential rotation between core and envelope in stars older than $\approx 100 \mathrm{Myr}$, Johnstone et al. (2015a) introduced the rigid body assumption into the magnetic braking torque formula derived by Watt et al. (2012), to estimate the evolution of rotation and winds. In this work, the solid body assumption is used to determine the mass and time dependence of the angular momentum and, thereon, develop very simple equations for the evolution of the Rossby number (a surrogate for magnetic activity), the torque, and the mass loss rate.

The product $J=I \Omega=2 \pi I / P$ - where $J$ is the angular momentum if the star is indeed rotating as a rigid body, $I$ is the moment of inertia of the whole star as given by the YaPSI model, $\Omega$ is the observed angular frequency and $P$ is the rotational period is plotted as a function of stellar mass in Figure 3. For the same reason as in Figure 2, only open cluster stars with errors less than $10 \%$ in their observational parameters are shown in this figure. Take notice on the following:

(i) The apparent or rigid body angular momentum of the vast majority of Kepler field stars lies in an area contained within well defined upper and lower limits, as long as $M / M_{\odot} \leq 1.2$.

(ii) There is a wide range of rigid body angular momenta among the least massive Pleiades and Praesepe stars, with no indication of a bimodal distribution. Rather than this, this dispersal conveys the impression that it is partly due to random initial conditions and times of birth. On the other hand, the possibility of a separate population of rapidly spinning low mass stars $\left(M / M_{\odot} \leq 0.7\right)$ is favored by the substantial number of fast rotators in the Praesepe cluster, where the imprint of initial conditions
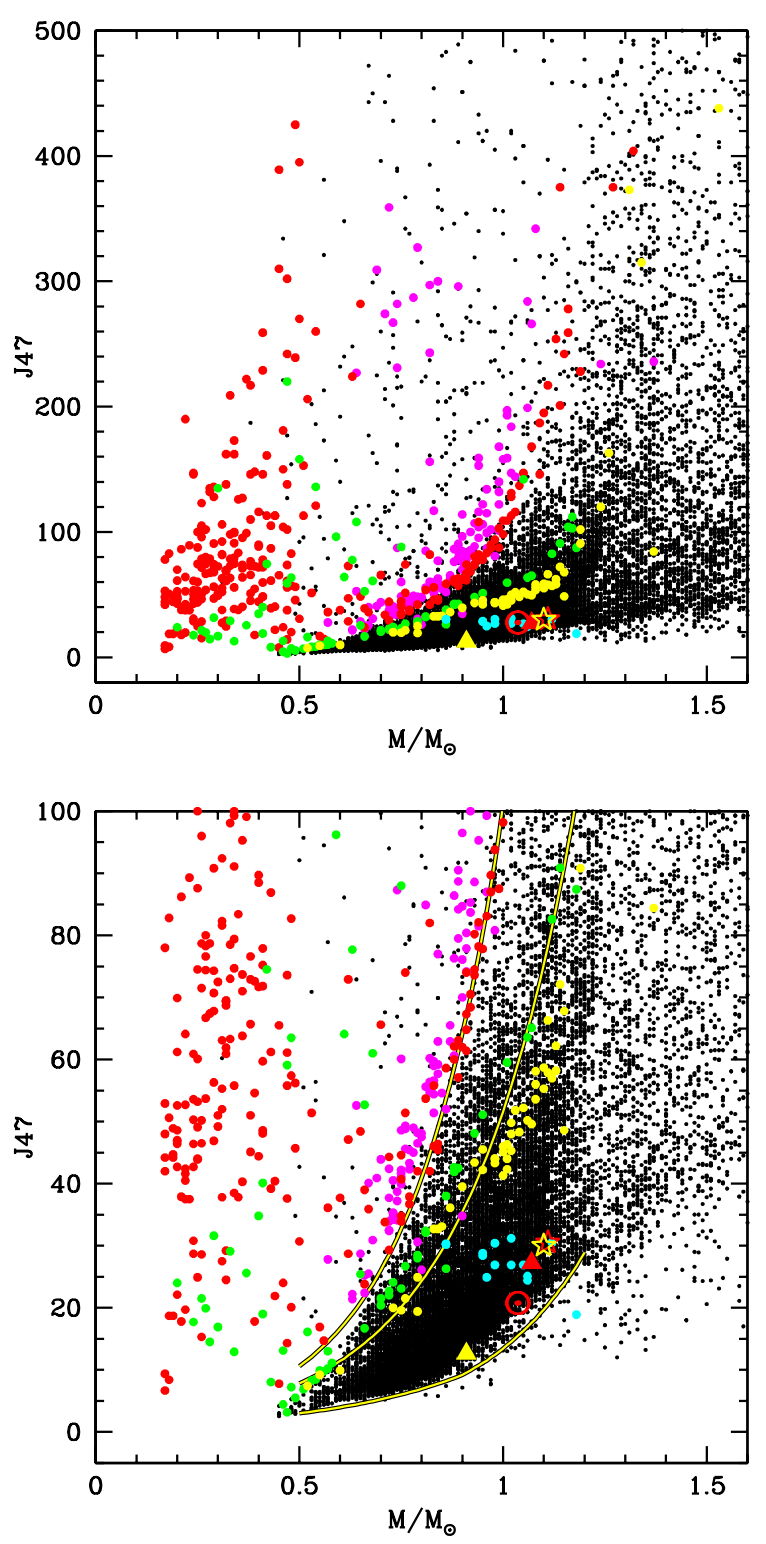

Fig. 3. Rigid body angular momentum (J47, $10^{47} \mathrm{gr} \mathrm{cm}^{-2} \mathrm{~s}^{-1}$ ) as a function of mass (solar units) in Kepler field (black), Pleiades (red, 120 Myr), M 35 (magenta, 155 Myr), Praesepe (green, 750 Myr), NGC 6811 (yellow, $1000 \mathrm{Myr}$ ), NGC 752 (yellow, $1350 \mathrm{Myr}$ ) and M 67 (cyan, 3500 Myr) stars. Only stars with errors of less than $10 \%$ in their observational parameters are shown in this figure. Stars and triangles are for $\alpha$ Cen A and B (yellow) and 16 Cygnus A and B (red). Yellow lines in the lower panel are rough approximations to a zero age rotational main sequence isochrone as defined by the Pleiades cluster (Equation 2), the $750 \mathrm{Myr}$ Praesepe isochrone (Equation 3) and the terminal main sequence rotational isochrone. (Equations 4 and 5). The color figure can be viewed online. 
is anticipated to be nearly gone. In any case, this dispersal is a major obstacle to an unambiguous age determination of stars less massive than $\approx 0.7 M_{\odot}$ and younger than $\approx 750 \mathrm{Myr}$.

(iii) The angular momentum tends to pile up in a decreasing range of values in the low mass range. This implies that age estimates based on rotational periods or angular momenta should be increasingly unreliable when the stellar mass is less than $\approx 0.6 M_{\odot}$. In addition, Epstein \& Pinsonneault (2014) observed that the inherent uncertainty of the initial conditions remains large for old stars below $0.6 M_{\odot}$.

(iv) For any given mass, the upper boundary (large angular momentum) of Kepler field stars coincides with the position defined by the Pleiades stars with the smallest angular momentum. Once again, it sets a well defined zero age rotational main sequence at $\approx 120 \mathrm{Myr}$. It is depicted by the upper yellow line shown in the lower panel of Figure 3, and can be roughly traced with the following equation if $0.5 \leq M / M_{\odot} \leq 1.2$,

$$
J_{\text {ple }}=1.19 \times 10^{47} 10^{1.953 M / M_{\odot}} \operatorname{gr~cm}{ }^{2} / \mathrm{s} .
$$

Notice that there is a factor of $\approx 100$ between the angular momentum of low and high mass stars, once they reach the zero age rotational main sequence.

(v) Quite evidently, notwithstanding the possibility of a rapidly spinning population, lower mass stars lose a larger fraction of their angular momentum before settling on the zero age rotational main sequence and take a longer time to do so.

(vi) An angular momentum sequence for slowly rotating stars from the Praesepe open cluster is also evident. It corresponds to the middle yellow line, and in the same mass range it is approximately given by,

$$
J_{\text {pre }}=1.18 \times 10^{47} 10^{1.640 M / M_{\odot}} \operatorname{gr~cm}{ }^{2} / \mathrm{s} .
$$

(vii) In contrast with the period distribution, a lower boundary for the angular momentum is very well defined by Kepler field stars. The terminal (as of today) main sequence rotational isochrone (bottom yellow line in the lower panel of Figure 3) is charted by the following equations,

$$
J_{\text {ter }}=2.94 \times 10^{46} 10^{1.661 \mathrm{M} / M_{\odot}} \mathrm{gr} \mathrm{cm}^{2} / \mathrm{s},
$$

if $0.9 \leq M / M_{\odot} \leq 1.2$, and

$$
J_{\text {ter }}=7.41 \times 10^{46} 10^{1.214 M / M_{\odot}} \mathrm{gr} \mathrm{cm}^{2} / \mathrm{s}
$$

if $0.5 \leq M / M_{\odot} \leq 0.9$.

The fact that less massive stars have not yet completed their main sequence rotational history is the likely reason why the slope changes at $M / M_{\odot} \simeq 0.9$.

(viii) As mentioned above, McQuillan, Mazeh \& Aigrain (2014) use gyrochronology models (Barnes, 2007; Mamajek \& Hillenbrand, 2008; Meibom et al. 2009) to set an age of 4500 Myr for stars occupying the lower boundary of the angular momentum (upper boundary for the period), but they conclude that these models under-predict stellar ages. A simpler and more intuitive estimate, is to set the timeline for this boundary considering that it must be defined by stars having nearly the same age as their main sequence lifetime $\left(\tau_{m s} \simeq 10^{4}\left(M / M_{\odot}\right)^{-2.5} \mathrm{Myr}\right)$ if $M / M_{\odot} \geq 0.9$, and somewhat less than the age of the Milky Way, say $\tau_{0}=13000 \mathrm{Myr}$, when $M / M_{\odot} \leq 0.9$ $\left(\tau_{0} \leq \tau_{m s}\right.$ in these low mass stars $)$.

(ix) For stellar masses between $\approx 0.9$ and $1.2 M_{\odot}$, the rigid body angular momentum of quite a few stars is less than the terminal value. If this is real, these stars may be leaving the main sequence. If so, they are expanding rapidly, their rotational shear and moment of inertia are escalating equally fast and, apparently, the most prominent star spots are spinning down by an even larger amount. Less massive stars are not doing so, since they are far from completing their main sequence lifetime.

Empirical relations between rigid body angular momentum and age for a specific mass can be found using either one or a combination of the Pleiades, Praesepe and terminal main sequence isochrones.

The simplest and more often used relation is an inverse square root law (a Skumanich-like relation). Choosing the Praesepe isochrone as the anchor line,

$$
J_{s q r}=J_{\text {pre }}(t / 750)^{-1 / 2},
$$

with $J_{\text {pre }}$ as given in Equation 3 and $t$ in Myr. Notice that this solution assumes a time invariant shape for the angular momentum distribution as a function of mass, in this case defined by the Praesepe open cluster stars. Comparing Equations 2 and 3, we can see that this assumption is not verified in the 
Pleiades star cluster, and we can expect poor results when Equation 6 is applied to stars that are somewhat younger than the Praesepe cluster. On the contrary, the similar shape of the Praesepe and terminal (for $\mathrm{M} / M_{\odot} \geq 0.9$ ) angular momentum distributions, implies that satisfactory results can be anticipated when Equation 6 is applied to older solar type stars.

Obviously, better adjustments valid for slowly rotating stars older than $120 \mathrm{Myr}$, can be obtained from three parameter solutions involving the three isochrones, such as the following exponential law,

$$
J_{e x p}=A \exp \left(-B t^{C}\right)
$$

where $t$ is in Myr, and $A, B$ and $C$ are mass dependent constants. These are given in Table 5 for a few selected masses. The table includes two e-folding times for the angular momentum, $\mathcal{T}_{0}$ and $\mathcal{T}_{120}$ (in Myr), i.e., the time it takes to reduce it by a factor equal to $1 / \mathrm{e}$ after the birth line, $t=0$, and after the age of the Pleiades, $t=120$. The e-folding time after the birth line may be deceptive, since there is no reason why we should expect that the power law approximation can be extrapolated to stellar ages that are less than $120 \mathrm{Myr}$. The e-folding time after the Pleiades rotational isochrone shows that the torque is increasingly vigorous in more massive stars. An interesting result is that after spending $\approx 13$ to $19 \%$ of their main sequence lifetime $(\approx 15 \%$ after discounting the Pleiades age), all 0.5 to $1.2 M_{\odot}$ main sequence stars loose $\approx 85 \%$ of the angular momentum they had when they were 120 Myr old.

Since a simpler exponential relation, $J=A \exp (B t)$, can be summoned for fast rotators with a saturated magnetic field (Chaboyer et al. 1995) and a fixed moment of inertia (Dennisenkov 2010; Amard et al. 2016), it is worth noticing that such a relation is completely at odds with the data when it is calibrated with the Pleiades and Praesepe isochrones. Thus, the stars defining these isochrones are not in the saturated regime and, obviously, are not fast rotators.

The mean and minimum stellar angular momenta found in the open clusters listed in Table 1 are displayed in Figures 4 and 5. By reason of insufficient data, stars less massive than $0.65 M_{\odot}$ are not considered. Since there is a limited amount of information for clusters older than $\approx 1000 \mathrm{Myr}$, main sequence stars with ages derived from asteroseismological data modeling are included. Listed in Table 6, some of these stars are binaries or host planetary systems (as the Sun, which is also included). The inverse square root and exponential relations between angular mo-
TABLE 5 EXPONENTIAL FITS*

\begin{tabular}{lcccrc}
\hline$M / M_{\odot}$ & $\mathrm{A}$ & $\mathrm{B}$ & $\mathrm{C}$ & $\mathcal{T}_{0}$ & $\mathcal{T}_{120}$ \\
\hline 0.50 & $1.62 \mathrm{e}+48$ & 0.105 & 0.293603 & 2157 & 7261 \\
0.60 & $3.19 \mathrm{e}+48$ & 0.200 & 0.247530 & 666 & 5091 \\
0.70 & $6.97 \mathrm{e}+48$ & 0.365 & 0.206752 & 131 & 3583 \\
0.80 & $1.50 \mathrm{e}+49$ & 0.540 & 0.183679 & 29 & 2675 \\
0.90 & $3.67 \mathrm{e}+49$ & 0.812 & 0.159828 & 3.7 & 2042 \\
1.00 & $7.56 \mathrm{e}+49$ & 0.949 & 0.157111 & 1.4 & 1563 \\
1.10 & $1.33 \mathrm{e}+50$ & 0.976 & 0.162967 & 1.2 & 1274 \\
1.20 & $2.08 \mathrm{e}+50$ & 0.919 & 0.175548 & 1.6 & 1075 \\
\hline
\end{tabular}

* Constants in exponential fits to the angular momentum, as given in Equation 7 . The e-folding times, $\mathcal{T}_{0}$ and $\mathcal{T}_{120}$ (both in Myr), are such that $B \mathcal{T}_{0}^{C}=-1$ and $B\left(\mathcal{T}_{120}^{C}-\right.$ $\left.120^{C}\right)=-1$.

mentum and age have been added to these figures (continuous line, equation 6 ; dotted line, equation 7 ).

These figures show that most of the angular momentum of all late type main sequence slow rotators, is lost during their first $\approx 500 \mathrm{Myr}$. This implies that the torque is exceptionally strong during this period. Thereon, the torque must be much more moderate, since angular momentum is lost in a very sedate fashion. As expected, the inverse square root relation defined by the Praesepe isochrone is inconsistent with the mean and minimum values of the angular momentum in clusters that are younger than $450 \mathrm{Myr}$. For these clusters, the exponential fit is more often than not compatible with the minimum value of the angular momentum. When $M / M_{\odot} \geq 0.9$ and the cluster age is larger than $\approx 450 \mathrm{Myr}$, both relations are consistent with the mean value of the angular momentum, and their difference is usually smaller than the observational uncertainties. This statement is also valid when stars are older than $750 \mathrm{Myr}$, and their mass is somewhat larger than $0.8 M_{\odot}$. With a couple of exceptions, these observations can be applied to stars with asteroseismological data.

According to equations 6 and 7 , the rigid body angular momentum of a $4500 \mathrm{Myr}$ solar mass star should be 2.1 and $2.2 \times 10^{48} \mathrm{gr} \mathrm{cm}^{-2} \mathrm{~s}^{-1}$, only $\approx 10 \%$ larger than the rigid body angular momentum of the Sun, $1.9 \times 10^{48} \mathrm{gr} \mathrm{cm}^{-2} \mathrm{~s}^{-1}$. Since scaling is implicitly included, the similarity with the power law fit is not surprising. On the other hand, the semblance with the inverse square root relation is suggestive, since no scaling is built into it. These equations can also be used to produce an age estimate using the rigid body angular momentum; in the solar case the result is 5500 and $5600 \mathrm{Myr}$. 
TABLE 6

STARS WITH ASTEROSEISMOLOGICAL DATA*

\begin{tabular}{lccccc}
\hline Name & Mass & Age & Period & I53 & J47 \\
\hline$\alpha$ Cen A & $1.10 \pm 0.01$ & $4850 \pm 500$ & $22.50 \pm 5.90$ & $9.29 \pm 0.20$ & $30.0_{-6.75}^{+53.1}$ \\
$\alpha$ Cen B & $0.91 \pm 0.01$ & $4850 \pm 500$ & $36.20 \pm 1.40$ & $6.25 \pm 0.02$ & $12.6_{-0.51}^{+0.55}$ \\
16 Cyg A & $1.11 \pm 0.02$ & $6800 \pm 400$ & $23.8_{-1.8}^{+1.5}$ & $9.98 \pm 0.72$ & $30.5_{-3.87}^{+2.73}$ \\
16 Cyg B & $1.07 \pm 0.02$ & $6800 \pm 400$ & $23.2_{-3.2}^{+11.5}$ & $8.68 \pm 0.44$ & $27.2_{-9.94}^{+5.94}$ \\
KIC 4914923 & $1.10 \pm 0.01$ & $6180 \pm 180$ & $20.49 \pm 0.00$ & $17.93_{-7.72}^{+35.0}$ & $63.6_{-27}^{+124.4}$ \\
KIC 6521045 & $1.04 \pm 0.02$ & $6240 \pm 640$ & $25.34 \pm 2.78$ & $8.63_{-1.07}^{+2.30}$ & $24.8_{-5.20}^{+10.4}$ \\
KIC 7871531 & $0.84 \pm 0.02$ & $9150 \pm 470$ & $33.72 \pm 2.60$ & $5.58_{-0.20}^{+0.20}$ & $12.0_{-1.20}^{+1.50}$ \\
KIC 8006161 & $1.04 \pm 0.02$ & $4690 \pm 530$ & $29.79 \pm 3.09$ & $6.32_{-0.28}^{+0.32}$ & $15.4_{-2.00}^{+2.70}$ \\
KIC 9955598 & $0.93 \pm 0.04$ & $6479 \pm 450$ & $34.75 \pm 6.31$ & $6.25_{-0.61}^{+0.72}$ & $13.1_{-3.10}^{+4.70}$ \\
KIC 10644253 & $1.13 \pm 0.05$ & $1070 \pm 250$ & $10.91 \pm 0.87$ & $7.94_{-0.24}^{+0.24}$ & $52.9_{-5.30}^{+6.40}$ \\
KIC $10963065^{p}$ & $1.07 \pm 0.03$ & $4260 \pm 400$ & $12.38 \pm 1.22$ & $35.1_{-22.9}^{+74.2}$ & $206_{-141}^{+506}$ \\
\hline
\end{tabular}

${ }^{*}$ Mass given in solar units, age in million years, period in days, I53 moment of inertia in $10^{53}$ gr $\mathrm{cm}^{2}$ and $\mathrm{J}_{47}$ angular momentum in $10^{47} \mathrm{gr} \mathrm{cm}^{2} \mathrm{~s}^{-1}$. Periods for $\alpha$ Cen A and B are from Bazot et al. (2007) and Dewarf, Datin \& Guinan (2010), and their mass and age are from Thévenin et al. (2002). Data for 16 Cygnus A and B, Davis et al. (2015). For the rest, the data is from Mathur et al. (2012), Metcalfe et al. (2014) and Ceillier et al. (2016). Superscript "p" is for stars with planets. Moment of inertia from YaPSI models, assuming $[\mathrm{Fe} / \mathrm{H}]=0.0$ and $Y_{0}=0.28$.

Thus, the rigid body angular momentum (as well as the rotational period) gives an indication of the evolutionary stage of any main sequence star, but the observational database shows that it can not be used to determine with any degree of precision the age of $\mathrm{F}$ and $\mathrm{G}$ main sequence stars younger than $\approx 450 \mathrm{Myr}$ or K type stars younger than $\approx 750 \mathrm{Myr}$. This limit is likely to be substantially higher for later spectral types. On the other hand, the exponential relation supported by the Pleiades, Praesepe and terminal isochrones for the rigid body angular momentum, or the simple inverse square root relation based on the Praesepe isochrone, may provide acceptable but not too precise estimates for the ages of older stars.

\subsection{Rotational Period as a Function of Mass and Time. Second Round}

The rigid body angular momentum depends on the detected rotational period and the unobserved moment of inertia $(J=2 \pi I / P)$. Since the moment of inertia is mass, metallicity and time dependent, the relation between rotational period and age should be more complicated.

The metallicity dependence of the moment of inertia is weak, but not insignificant. For otherwise identical conditions, higher metallicities produce larger moments of inertia, with an up to $\approx 10 \%$ difference between the highest $\left([\mathrm{Fe} / \mathrm{H}]=0.3, Y_{0}=\right.$ $0.31)$ and lowest $\left([\mathrm{Fe} / \mathrm{H}]=-0.5, Y_{0}=0.25\right)$ values considered by YaPSI, the gap being smaller for lower mass stars.

As mentioned before, the time dependence of the moment of inertia can be substantially more important. The evolution of the moment of inertia of $0.5-$ $1.2 M_{\odot}$ stars with a solar composition was depicted in Figure 1. It shows that it declines very rapidly at the beginning of the main sequence. After $100 \mathrm{Myr}$ or so it settles at a relatively stable value that lasts until roughly half the main sequence lifetime. During this stage, the moment of inertia of $\approx 0.4$ to $1.2 M_{\odot}$ stars is approximately equal to its $1000 \mathrm{Myr}$ old value. YaPSI models for $1000 \mathrm{Myr}$ old stars with $[\mathrm{Fe} / \mathrm{H}]=0.0$ and $Y_{0}=0.28$, lead to the following fit to the moment of inertia for a stellar mass range between 0.5 and $1.2 M_{\odot}$,

$$
I \simeq 6.99 \times 10^{52} 10^{0.965} \mathrm{M} / M_{\odot} \mathrm{gr} \mathrm{cm}^{2} .
$$

The regression coefficient and the square root of the mean square error are equal to 0.98 and 0.09 . The metallicity effect may add an additional uncertainty of $\approx 5 \%$ in the high mass limit, less than this in lower mass stars. For a one solar mass star, the moment of inertia predicted by this Equation is $6.45 \times 10^{53} \mathrm{gr} \mathrm{cm}^{2}$, somewhat less than the precise value, $6.71 \times 10^{53} \mathrm{gr} \mathrm{cm}^{2}$, and appreciably smaller than the moment of inertia of a $4500 \mathrm{Myr}$ old one solar mass star, $6.95 \times 10^{53} \mathrm{gr} \mathrm{cm}^{2}$.

Equation 8 and the inverse square root relation between angular momentum and age lead to the fol- 

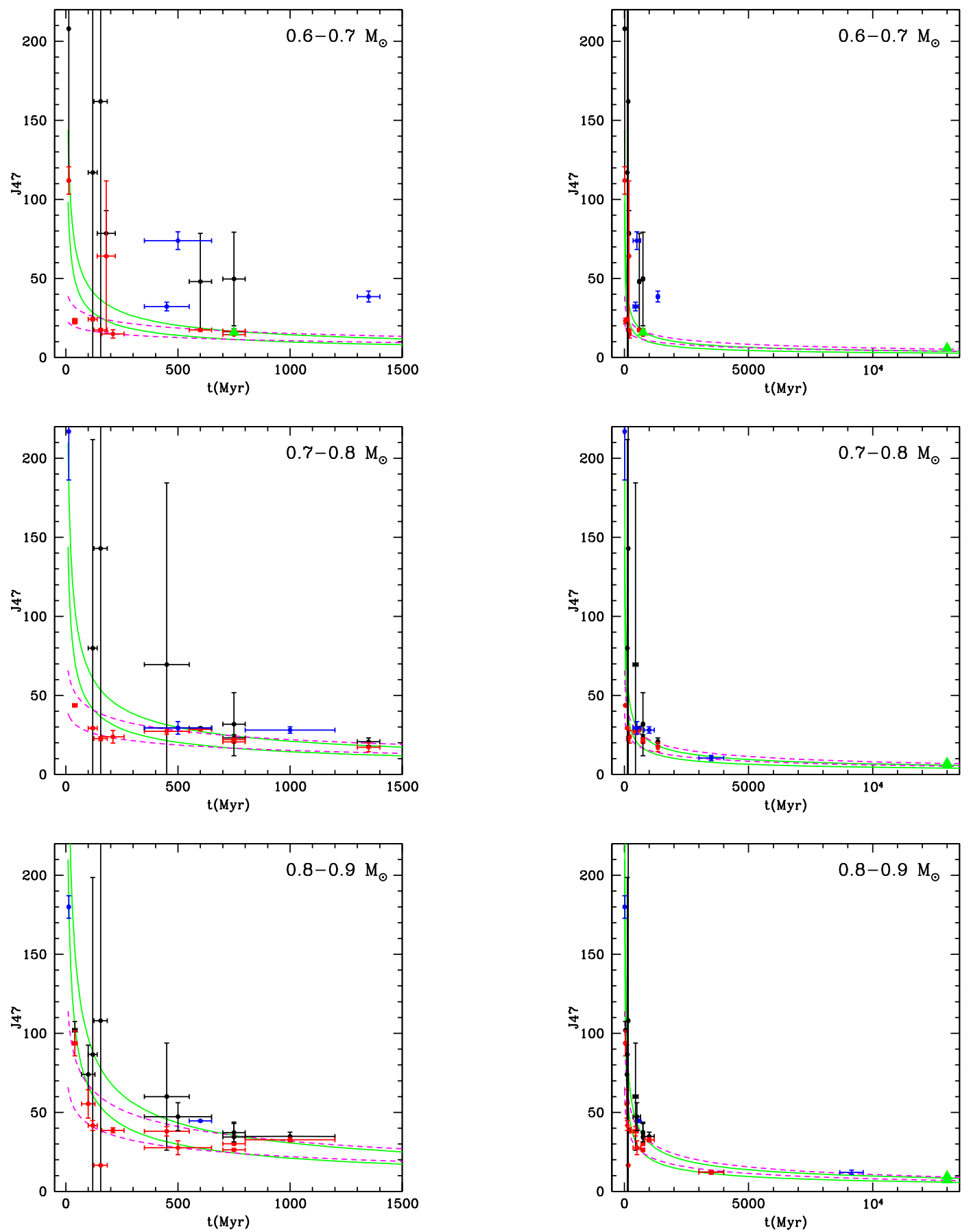

Fig. 4. Rigid body angular momentum $\left(\mathrm{J} 47,10^{47} \mathrm{gr} \mathrm{cm}^{2} \mathrm{~s}^{-1}\right)$ as a function of time for stellar masses between 0.6 and $1.2 M_{\odot}$ in $0.1 M_{\odot}$ intervals. Mean and minimum angular momentum values for the open cluster stars listed in Table 1 are shown in black and red. Single stars from a cluster or from the group of stars listed in Table 6 are shown in blue. The Sun is represented with a red circle and the green triangles in the right hand side figures stand for the angular momentum of the terminal rotational main sequence. The continuous and dotted lines are the inverse square root (green) and exponential fits (magenta) to the angular momentum of the lower and upper mass limits written at the top right hand side of these figures. The color figure can be viewed online. 

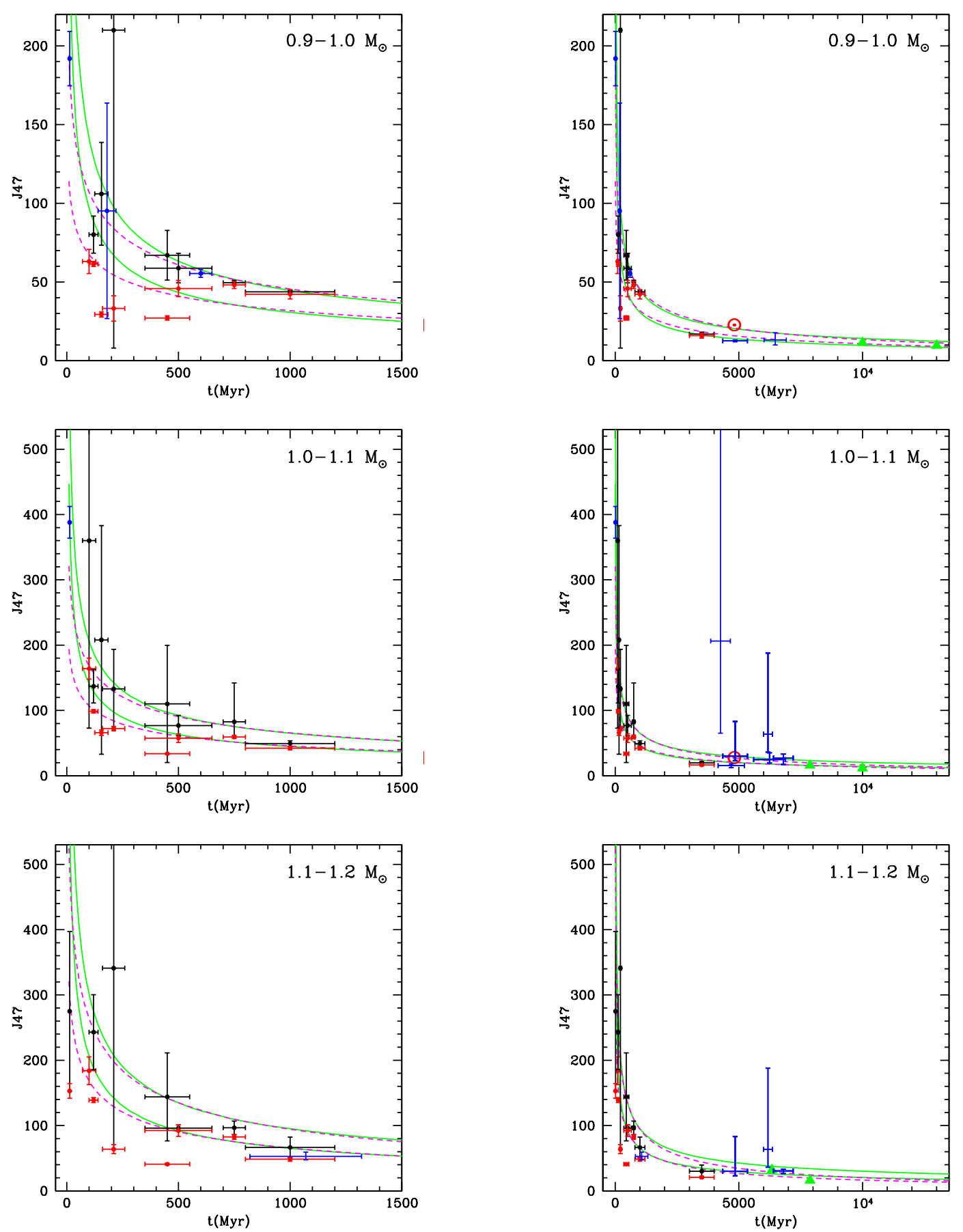

Fig. 5. Same caption as in Figure 4. The color figure can be viewed online.

lowing direct connection between age and rotational period during the time span where the moment of inertia is approximately constant,

$$
t \simeq 0.40410^{1.35} M / M_{\odot} P_{d}^{2} \mathrm{Myr} .
$$

This equation returns a disappointing age of $6600 \mathrm{Myr}$ for the Sun. The exponential fit leads to a more intricate relationship, but gives the same age for our star, i.e., $\approx 1000 \mathrm{Myr}$ more than the age estimate based on the rigid body angular momentum.

In the second half of their main sequence lifetime, stars build an increasingly larger moment of inertia and the mean rotational period will increase even 
in the absence of rotational braking. Notice that the consequence on surface rotation may be more pronounced, since differential rotation may result as the core contracts and spins up and the envelope expands and slows down. These effects are still insignificant in lighter stars, but gain in importance in $1.2,1.1,1.0$ and $0.9 M_{\odot}$ stars as soon as they are $\approx 1400,3600,4500$ and 7900 Myr old. For instance, without rotational braking, the solar period will be around 32,38 and 68 days when our star is 8000,9000 and $10000 \mathrm{Myr}$ old. The rotational period would be equal to 38,47 and 90 days if the evolution of angular momentum resembles the inverse square root relation (Equation 6) or 39, 49 and 95 days if it is described by the exponential law (Equation 7). Since the longest rotational period of the one solar mass stars included in this paper is 63 days, these numbers suggest that other drivers of rotational braking (mass loss, magnetic field intensity, wind acceleration) are secondary agents in the evolution of stellar periods during the second half of the main sequence. Actually, there may be some evidence for inefficient magnetic braking in the Sun, where the high latitude wind was found to be superAlfvenic close to the solar surface (McComas et al. 2000).

\subsection{Magnetic Activity as a Function of Mass and Time}

Stellar dynamos sustain poloidal-toroidal magnetic fields (the $\alpha-\Omega$ mechanism) as long as Coriolis forces dominate inertial forces within the convective region (Durney \& Latour 1978). The relation between these forces is quantified with the Rossby number, which can be defined as

$$
R_{o}=P / \tau_{c}=2 \pi I / \tau_{c} J,
$$

where $\tau_{c}$ is the convective turnover time.

A strong argument for the conjecture that the magnetic field of main sequence stars with a convective envelope is produced by the $\alpha-\Omega$ dynamo mechanism is based on the observation that the X-ray and chromospheric to bolometric luminosity ratios are proportional to $R_{o}^{-2}$ in the non-saturated regime (Noyes et al. 1984; Pizzolato et al. 2003). This thesis has been disputed by Reiner, Schüssler \& Passegger (2014), arguing that these relations hold if the convective overturn time scales as $L_{b o l}^{-1 / 2}$. Since both quantities are roughly constant during most of main sequence, this proportionality should be true for any star during this relatively stable time period. On the other hand, the proportionality constant may depend on the stellar mass. Interestingly, during this stable phase $L_{b o l}^{1 / 2} \times \tau_{c} \simeq 30\left(L_{b o l}\right.$ in solar units, $\tau_{c}$ in days) if $0.4 \leq M / M_{\odot} \leq 1$, close to 20 if $M / M_{\odot}=1.1$ and around 7 when $M / M_{\odot}=1.2$. Admittedly, the convective turnover time provided by YAPSI is associated to the tachocline, not to the position where the dynamo is more effective. Even so, these numbers suggest that the Parker dynamo mechanism has an effect on magnetic braking, at least in non-fully convective stars less than, or as massive as, the Sun. Whichever the case, Reiner et al. (2014) rightly emphasize the need to explore and consider a wider range of mechanisms for the generation of magnetic fields in low mass main sequence stars.

Thus, assuming that the $\alpha-\Omega$ mechanism is operative, magnetic activity and rotational braking will tend to be suppressed when the Rossby number is large. It has been argued that this explains the existence of anomalously rapid rotation in some old field stars (van Saders et al. 2016), and it may be the reason why KIC 4914923, KIC 5184732 and KIC 10963065 have such an atypically large angular momentum (see Table 6).

The value of the convective turnover time depends on the convection parameter (usually taken as equal to the solar value, $\alpha=1.875)$ and the assumed depth of the region where the dynamo is being generated. Under the same circumstances, lengthier convection times and smaller Rossby numbers are obviously associated to dynamos running closer to the tachocline but, except for the scale difference, the mass and time dependence of the convective turnover time does not seem to be affected by this supposition (Landin, Mendes \& Vaz 2010).

According to YaPSI models with solar-like composition $\left([\mathrm{Fe} / \mathrm{H}]=0.0\right.$ and $\left.Y_{0}=0.28\right)$, the convective turnover time of all late type main sequence stars is equal to a few hundred days during the first 10 to $100 \mathrm{Myr}$. Later on, it remains roughly constant, almost up to the end of main sequence. Consequently, as stars spin down during their main sequence evolution, the Rossby number will increase, the $\alpha-\Omega$ mechanism will weaken and magnetic braking will become increasingly inefficient. Since the convective turnover time during this stage is $\geq 100$ days when $M \leq 0.6 M_{\odot}$, and $\approx 70,55,40,30,15$ and 5 days when $M=0.7,0.8,0.9,1.0,1.1$ and $1.2 M_{\odot}$, the demise of the dynamo effect may begin significantly earlier in stars with shallow convective regions.

The history of the Rossby number for 0.5 to $1.2 M_{\odot}$ stars was determined using the exponential and inverse square root approximations for the evolution of the angular momentum (similar results are 
obtained). In these calculations the Rossby number of a $4500 \mathrm{Myr}$ old one solar mass star is equal to 0.87 . This is significantly less than an often quoted value (2.16 in van Saders et al. 2016) but, as discussed above, part of this difference is probably related to the assumed depth of the dynamo producing region. Thus, depending on the precise definition of the convective turnover time, either one of these numbers (both close to one) can be taken as the Rossby number where magnetic activity and rotational braking may begin to decline.

The evolution of the Rossby number is shown in Figure 6. Notice that $R_{o} \geq 1$ for 1.2, 1.1, 1.0 and $0.9 M_{\odot}$ stars once they are older than $\approx 500,2500$, 5500, and $9500 \mathrm{Myr}$. In 1.2 and $1.1 M_{\odot}$ stars, the cessation of the dynamo mechanism may occur before there is a significant change in their moment of inertia. With the un-anticipated exception of $1.2 M_{\odot}$ stars, the possible disruption of the magnetic dynamo occurs much later than the $\mathcal{T}_{120}$ e-folding times for the angular momentum.

Kitchainov \& Nepomnyashchikh (2017) had noticed that the interruption of large scale dynamos may be the reason why gyrochronology fails to predict the age of older stars. If this is correct, their surviving magnetic fossil field may be the main rotational braking source during the time interval between the fading $\alpha-\Omega$ mechanism and the swelling moment of inertia. Later on, the transformation of the moment of inertia will eventually determine the evolution of the rotational period. In less massive stars, dynamo activity will decline at the same time as the moment of inertia escalates, and their continuously changing relative importance will impress the history of the rotational period during the second half of the main sequence stage.

\subsection{Torque as a Function of Mass and Time}

Differences in angular momentum between pairs of isochrones with respect to the youngest of the two, are displayed on the left hand side of Figure 7 (associated formulas are shown in this figure). The corresponding mean loss rates per Gyr are shown on the right hand side.

Close to $60 \%$ and $25 \%$ of the initial rigid body angular momentum of 1.2 and $0.5 M_{\odot}$ slowly rotating stars is lost during the few hundred million years separating the Pleiades and Praesepe isochrones. At this stage, the mean loss rate is around $90 \%$ and a bit more than $40 \%$ per Gyr for 1.2 and $0.5 M_{\odot}$ stars. Loss rates are much smaller after $\approx 750 \mathrm{Myr}$. Near the end of their main sequence lifetime, $88 \%$ and $86 \%$ of the initial angular momentum of 1.2 and

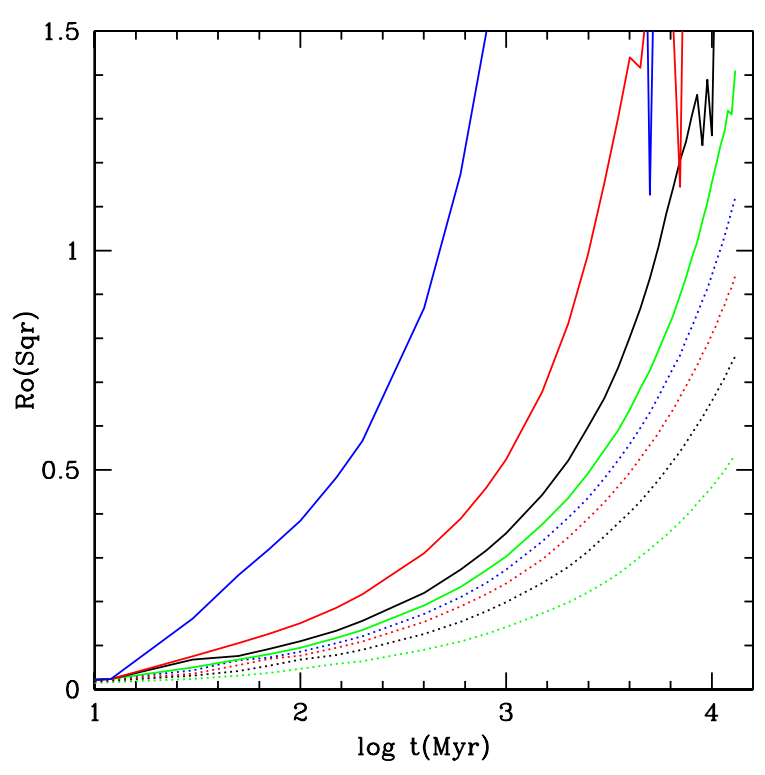

Fig. 6. Evolution of the Rossby number during the main sequence stage, assuming that the angular momentum is as given in equation 6. Dotted lines stand for $M / M_{\odot}=0.5$ (green), 0.6 (black), 0.7 (red) and 0.8 (blue). Continuous lines stand for $M / M_{\odot}=0.9$ (green), 1.0 (black), 1.1 (red) and 1.2 (blue). The color figure can be viewed online.

$0.9 M_{\odot}$ stars has been lost. At this point in time, the oldest 0.7 and $0.5 M_{\odot}$ stars have lost $80 \%$ and $73 \%$ of the angular momentum they had when landing on the Pleiades isochrone. From these two figures, it is once again clear that the relative and absolute rigid body angular momentum loss rates are always appreciably larger in shorter-lived, more massive, stars.

The inverse square root and exponential fits to angular momentum evolution (Equations 6 and 7), lead to the following expressions for the angular momentum loss rates (i.e., the torque $\tau=d J / d t$ ) acting on slowly rotating late type main sequence stars older than approximately $120 \mathrm{Myr}$,

$$
\begin{aligned}
& \tau_{s q r}=-J_{\text {pre }} 750^{1 / 2} t^{-3 / 2} / 2 \text {, } \\
& \tau_{s q r}=-1.62 \times 10^{48} 10^{1.640 M / M_{\odot}} t^{-3 / 2} \\
& \mathrm{gr} \mathrm{cm}^{2} \mathrm{~s}^{-1} \mathrm{Myr}^{-1} \text {, }
\end{aligned}
$$

$\tau_{\text {exp }}=A B C \exp \left(B t^{C}\right) t^{C-1} \quad \mathrm{gr} \mathrm{cm}^{2} \mathrm{~s}^{-1} \mathrm{Myr}^{-1}$,

with $t$ in Myr, and constants $A, B$ and $C$ as given in Table 5. Notice that in both cases the torque can be re-written in the following way

$$
\tau=K J t^{\gamma}=K I \Omega t^{\gamma}
$$



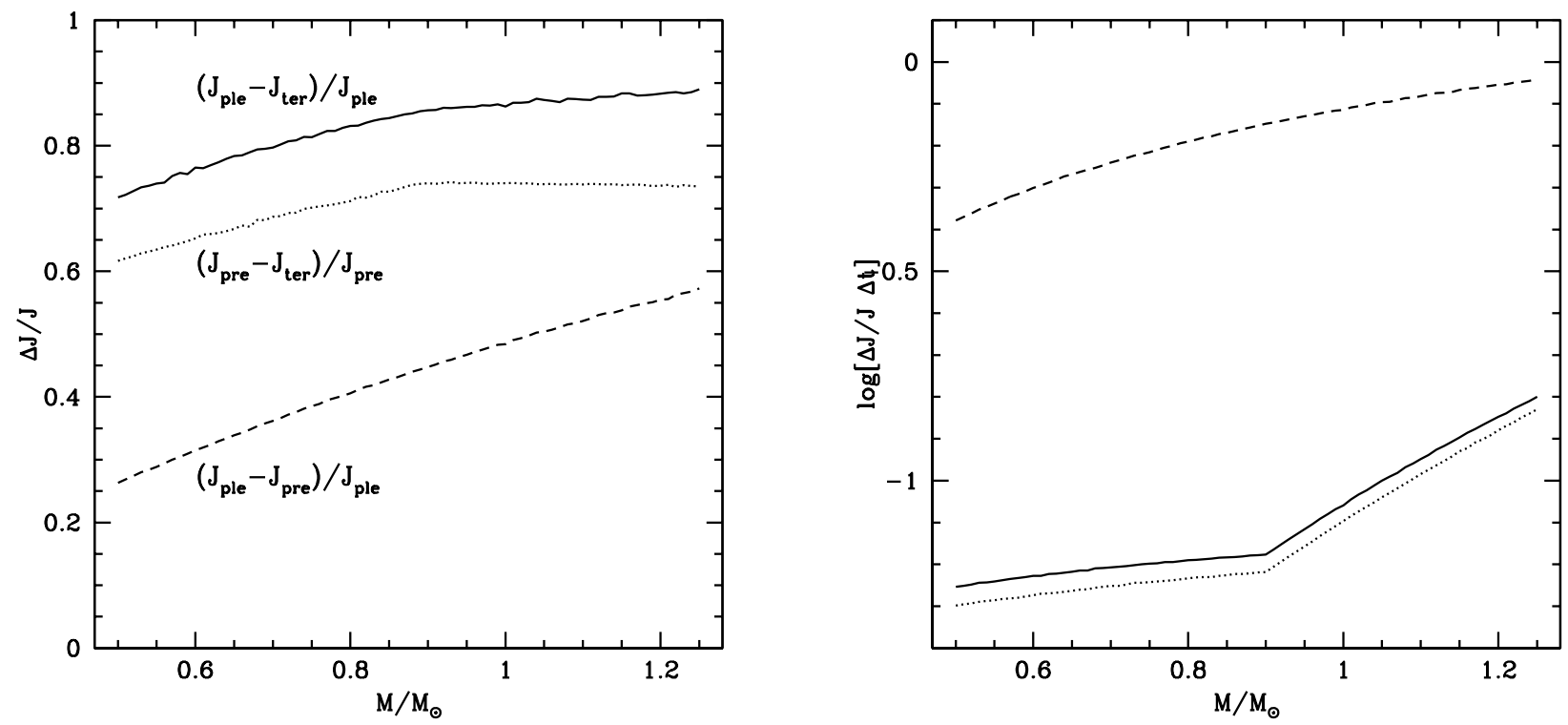

Fig. 7. On the left, differences in the angular momentum between the Pleiades, Praesepe and terminal isochrones, relative to the angular momentum of the younger one $(\Delta J / J)$. On the right, logarithm of the mean relative angular momentum loss rate per Gyr, $\log (\Delta J / / J \Delta t)$, for the time interval between the Pleiades and terminal isochrone (continuous line), the Praesepe and terminal isochrones (dotted line) and the Praesepe and Pleiades isochrones (short-dashed line).

with $K=-1 / 2$ and $\gamma=-1$ in the inverse square root relation, and $K=-B C$ and $\gamma=C-1$ in the exponential approximation.

These torques are usually smaller than those where the radiative core is spinning faster than the envelope, since there is less angular momentum when the entire star rotates at the surface rate. This can be visualized in Figure 8. Beyond $100 \mathrm{Myr}$, both of them are up to a factor of 5 smaller than the torques produced by a couple of models for the slowly rotating branch of one solar mass stars, where solid body rotation is not assumed and angular momentum is transferred from the radiative core to the convective envelope in two different ways (Amard et al. 2016).

The temporal evolution of the absolute value of the torque under the inverse square root and exponential approximations is plotted in Figure 9. As can be seen, there is a conspicuous difference between the inverse square root and exponential approximations when stars are younger than a few hundred million years. This disparity extends for a longer time and is more pronounced in low mass stars. Since an inverse square root law is at odds with the observed evolution of angular momentum in young stars, particularly young low mass stars (see $\S 3.2$ ), it follows that equation 11 cannot be a close description of the torques applied to these objects. On the other hand, the exponential fit is a reasonably close approxima-

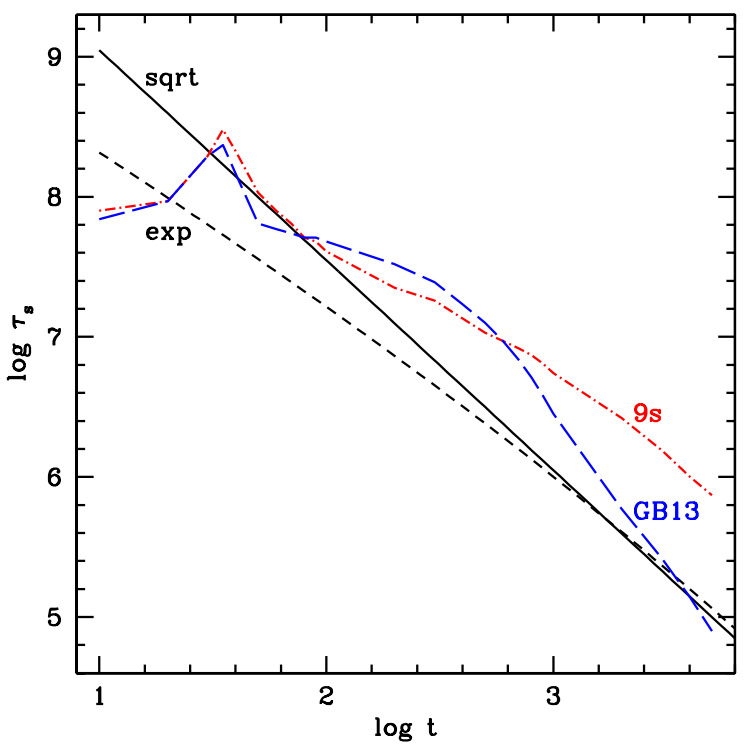

Fig. 8. Absolute value of the specific torque per year, $\tau_{s}$, as a function of time (Myr), for a stellar mass equal to $1.0 M_{\odot}$. The continuous (sqrt) and dotted (exp) lines are the inverse square root and exponential fits as given by equations 11 and 12 . The blue (GB13) and red (9s) tracks are from Amard et al. (2016). The color figure can be viewed online. 

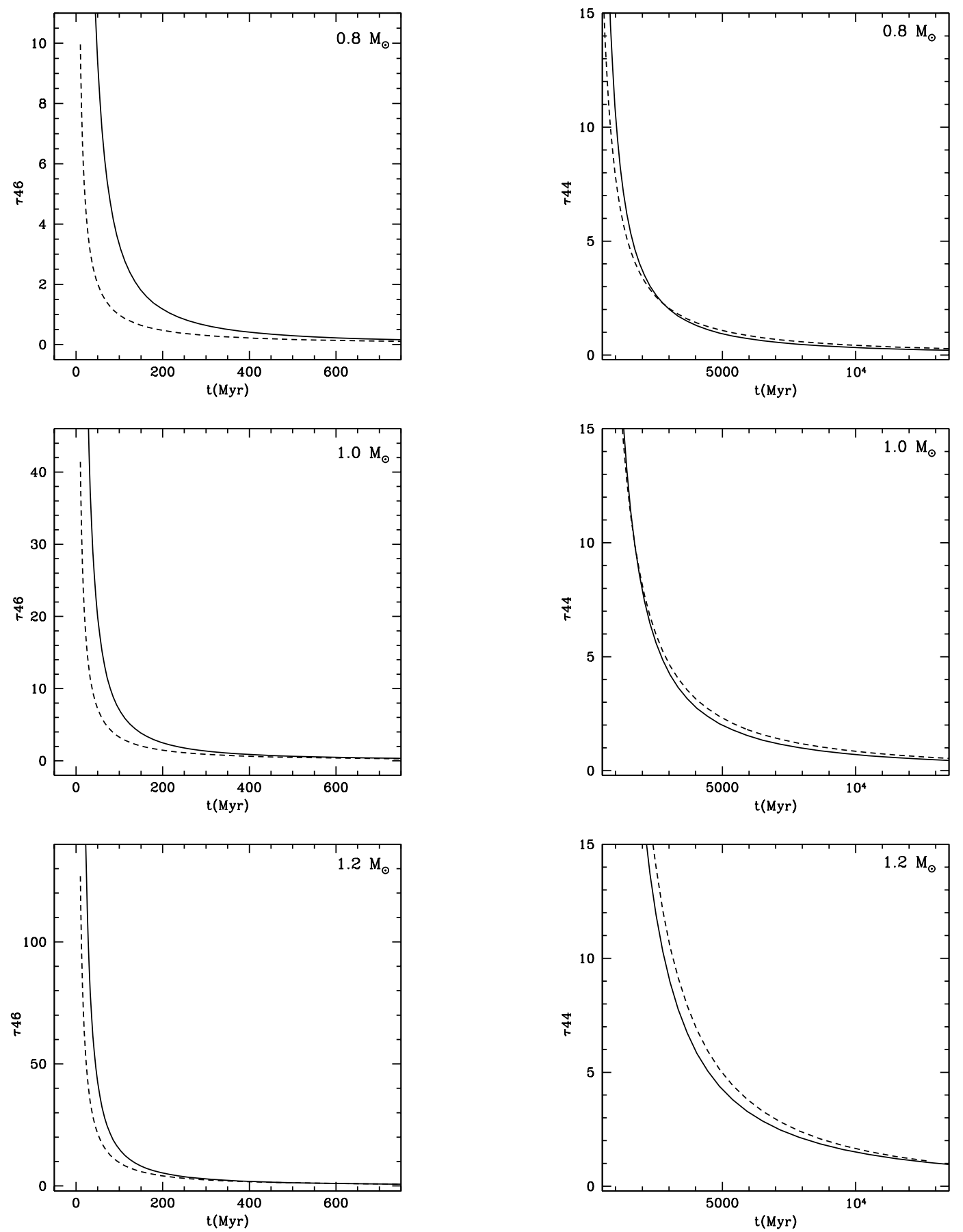

Fig. 9. Absolute value of the torque in units of $10^{46}$ and $10^{44} \mathrm{gr} \mathrm{cm}^{2} \mathrm{~s}^{-1} \mathrm{Myr}^{-1}(\tau 46$ and $\tau 44)$ as a function of time, for stellar masses equal to $0.8,1.0$ and $1.2 M_{\odot}$. The upper age limit on the left hand side figures is $750 \mathrm{Myr}$, and the lower age limit on the right hand side figures is $500 \mathrm{Myr}$. The continuous and dotted lines are torques produced by the inverse square root and exponential fits to the evolution of the angular momentum (equations 11 and 12).

tion to the angular momentum evolution of young stars, so it should lead to a more accurate depiction of the real torque for slowly rotating stars that are at least as old as the Pleiades cluster, as long as radial differential rotation is nearly absent. Notice that the magnitude of the torque at any given age is 
much smaller when the exponential fit is used, and that the demands imposed by a smaller torque are more easily met.

Figure 9 also confirms that the torque is very powerful and variable at least during the first $\approx 500 \mathrm{Myr}$. After dropping by a couple of orders of magnitude, it is increasingly weaker and stable. Thus, it seems that at least one of the agents driving angular momentum losses, be it the magnetic field strength and/or the mass loss rate, is extremely robust and mutable during the early stages of main sequence evolution. Later on, when the torque is less effective and variable, at least one of these agents must be considerably more stable and moderate. Notice that after $\approx 500 \mathrm{Myr}$, the torques inferred from inverse square root and exponential relations for the angular momentum are almost indistinguishable.

\subsection{A Simple Model for the Torque and Mass Loss Rate as a Function of Time}

These empirical approximations and an idealized model of the torque, can be used to explore the mass loss rate as stars evolve during the main sequence. At any given point and time, the torque is given by

$$
\tau=\dot{M} \Omega R_{c}^{2},
$$

where $\dot{M}$ is the mass loss rate, $\Omega$ is the rotational frequency and the lever arm $R_{c}$ - known as the corotation or Alfven radius - is the distance between the stellar surface and the point where angular momentum is being lost. The co-rotation radius is determined from the proposition that angular momentum is lost when the stellar wind is detached from the magnetic field. This happens when fluid pressure equals magnetic pressure,

$$
\rho V^{2}=\rho V R_{c} \Omega=\dot{M} \Omega / 4 \pi R_{c}=B_{c}^{2} / 4 \pi,
$$

where $B_{c}$ is the magnetic flux density at $R_{c}$. Since the mass loss rate and the magnetic field strength are latitude, longitude and time dependent, and the angular velocity is latitude and time dependent, it follows that the co-rotation radius cannot be uniform and stable. Thus, a continuously changing crumpled ball of paper - definitely not a smooth and steady regular figure - is a plausible visual representation of the co-rotation surface,

The detailed configuration of the magnetic field above the stellar surface has long been recognized as a very difficult problem (Mestel 1968), since it is an extremely complex mixture of small, medium and large scale dynamic structures rising throughout the convective envelope (e.g., Lang et al. 2014).
But it is worth remembering that the dipole is the constituent with the slowest radial decay and therefore presides over the strength of the magnetic field at the co-rotation radius, as was shown by Finley \& Matt (2018) after analyzing the combined effect that dipolar, quadrupolar and octupolar geometries had on the magnetic braking mechanism.

In the present day Sun, magnetic fields are produced close to the tachocline, the thin layer between the radiative core and the convective envelope (Charbonneau 2010). But different processes are involved in other stars. In fully convective stars, magnetic fields cannot be generated by the classical $\alpha-\Omega$ process, and it is possible that small scale magnetic fields are produced at various depths by turbulent velocity fields (Durney, De Young \& Roxburgh 1993). Additionally, strong toroidal azimuthal fields can show up directly at the stellar surface of stars with a mass between 0.1 and $1.5 M_{\odot}$, and it has been suggested that these are produced by dynamos distributed throughout the convection zone (Donati et al. 1992; Donati \& Collier Cameron 1997; See et al. 2015), an idea supported by Brown et al. (2010). Thus, different magnetic energy sources can be located relatively close to the stellar surface but not beyond the tachocline (if there is one).

An extreme, manageable and sensible simplification is to assume that the magnetic field is produced by a collection of dipoles placed at various distances $h$ under the stellar surface. If $h$ is much larger than the physical size of each dipole,

$$
B_{c}=B_{s} h^{3} /\left(h+R_{c}\right)^{3},
$$

where $B_{s}$ is the dipole's magnetic field density emerging at the stellar surface. Magnetic braking is effective if the co-rotation radius is at least a few stellar radii from the surface, so that it is quite likely that $h / R_{c}<0.01$. If this is so, and the stellar surface is covered by any number of magnetic active regions produced by identical dipolar fields, the total torque is

$$
\tau \simeq\left(f_{s} B_{s}\right)^{4 / 5} h^{12 / 5} \dot{M}^{3 / 5} \Omega^{3 / 5},
$$

where the filling factor, $f_{s}$, is the area covered by the regions that contribute to rotational braking, over the stellar surface area. Notice that the surface field, the mass loss rate and the angular velocity $\left(B_{s}, \dot{M}\right.$ and $\left.\Omega\right)$ are averages of these quantities over a time period where all longitudinal and latitudinal fluctuations have been smoothed out, i.e., the twisted short-time dependent co-rotation surface has been idealized as a smooth long-time dependent spherical figure. This implies that the long-time period must comprise several stellar cycles and, even 
so, it is almost certain that the distance between the stellar and co-rotation surfaces depends on latitude. Consequently, these averages cannot be expected to be equal or similar to values obtained from observations, since these are completed in shorter time spans.

Mean values of the total field strength can be obtained by measuring Zeeman splitting of unpolarized spectral lines, a procedure known as the ZB technique. This method provides no information on the magnetic field geometry, but it includes the contribution of magnetic field structures of all magnitudes and sizes. Notice that some of these structures may not participate in the magnetic braking process. With a data base of close to a couple of dozen late type main sequence stars, Saar $(1996,2001)$ found that, except for the most active, their magnetic field density was close to the photospheric equipartition value (same magnetic and thermal pressures), $f_{s} B_{s}$ is between $\approx 20$ and $4000 \mathrm{G}$ and $f_{s} B_{s}$ is nearly proportional to $\Omega^{5 / 3}$ if the rotational period is more than 3 days (the reported exponent is 1.7). The data show that there is an almost linear relation between the X-ray flux and $f_{s} B_{s}$, but there is no mention of possible correlations between magnetic field and age or spectral type.

The ZDI technique has been applied to Stokes $V$ profiles, but in this case the magnetic field flux density, $\left\langle\left|B_{V}\right|\right\rangle$, does not include the longitudinal component and small scale fields. Using this technique, Vidotto et al. (2014) calculated the large scale surface magnetic field densities of some 60 main sequence stars, and found that $\left\langle\left|B_{V}\right|\right\rangle$ is no larger than $\approx 100 \mathrm{G}$ when the spectral type is between $\mathrm{F} 7$ and M3, but larger than this and up to $1580 \mathrm{G}$ in later spectral types. From a sample of close to 60 stars, they worked out that $\left\langle\left|B_{V}\right|\right\rangle$ is nearly proportional to $\Omega^{4 / 3}$ (their exponent is 1.32 ), though there is a very large scatter and the correlation coefficient is not particularly good. Using age estimates based on different methods, they conclude that $\left\langle\left|B_{V}\right|\right\rangle \alpha t^{-0.655}$. They also suggest that small and large scale fields could share the same dynamo generation process, but no mention is made of a detailed connection between spectral type and magnetic field in main sequence stars.

Taking these relations between magnetic field and angular velocity into account,

$$
\tau \alpha h^{12 / 5} \Omega^{(4 \beta+3) / 5} \dot{M}^{3 / 5},
$$

with $\beta \simeq 4 / 3$ for the global field (ZDI technique) or $5 / 3$ when all magnetic structures are included (ZB technique). Combining this with equation 13 for the torque, the evolution of the mass loss rate is given by

$$
\dot{M}(M, t)=A(M) I^{(1-4 \beta) / 3} t^{5 \gamma / 3} J^{(2-4 \beta) / 3} h^{-4},
$$

where $A(M)$ is a mass dependent function.

Two solutions for the ratio of the mass loss rate at any time, with respect to the mass loss rate at $t=100 \mathrm{Myr}, \dot{M}(100)$, are displayed in Figure 10 . These figures are for $\beta=5 / 3$ (very similar figures are obtained when $\beta=4 / 3$ ). Being a much better approximation to the evolution of angular momentum during the entire main sequence, the exponential fit (equation 7, $\gamma=C-1$ and $C$ as given in Table 5) was used to compute the mass loss rate. The moment of inertia is taken from YaPSI models with $[\mathrm{Fe} / \mathrm{H}]=0.0$ and $Y_{0}=0.28$. On the left hand side, the average depth of the magnetic field source $(h)$ is assumed to be constant in time, and is therefore included in the empirical mass dependent function $A(M)$. On the right hand side, $h$ does change in time but not the ratio $h / h_{t}$, where $h_{t}$ is depth of the convective envelope. In this case, $h / h_{t}$ is included in $A(M)$ and the depth of the convective envelope is an additional mass and time dependent parameter. As the moment of inertia, $h_{t}$ is taken from YaPSi models with $[\mathrm{Fe} / \mathrm{H}]=0.0$ and $Y_{0}=0.28$.

If this simple model for the torque is basically correct and the magnetic field behaves as has been discussed, these figures call upon these outcomes:

(i) After $\approx 100 \mathrm{Myr}$, the mass loss rate functions of all $0.5-1.2 M_{\odot}$ slowly rotating main sequence stars converge to a time dependence that is approximately proportional to $t^{-1}$. After $\approx 750 \mathrm{Myr}$, the inverse square root approximation for the angular momentum can be used to describe the torque and it is easy to see that $\dot{M}$ is proportional to $t^{-8 / 9}$ if $\beta=5 / 3$, and to $t^{-10 / 9}$ if $\beta=4 / 3$. This time dependence lasts up to the first half of the main sequence lifetime of these stars, i.e., as long as there are no major alterations in the moment of inertia and the depth of the magnetic field source. Other authors obtained very similar time dependencies for the mass loss rate of inactive $1 M_{\odot}$ stars: $t^{-1.1}$ for ages between 200 and $7000 \mathrm{Myr}$ (Cranmer \& Saar 2011), $t^{-1.23}$ (Suzuki et al. 2013) and $t^{-0.75}$ (Johnstone et al. 2015a) for stars older than 700 Myr.

(ii) If the mean physical depth of magnetic field sources is constant in time, mass loss rates of all spectral types increase during the first 

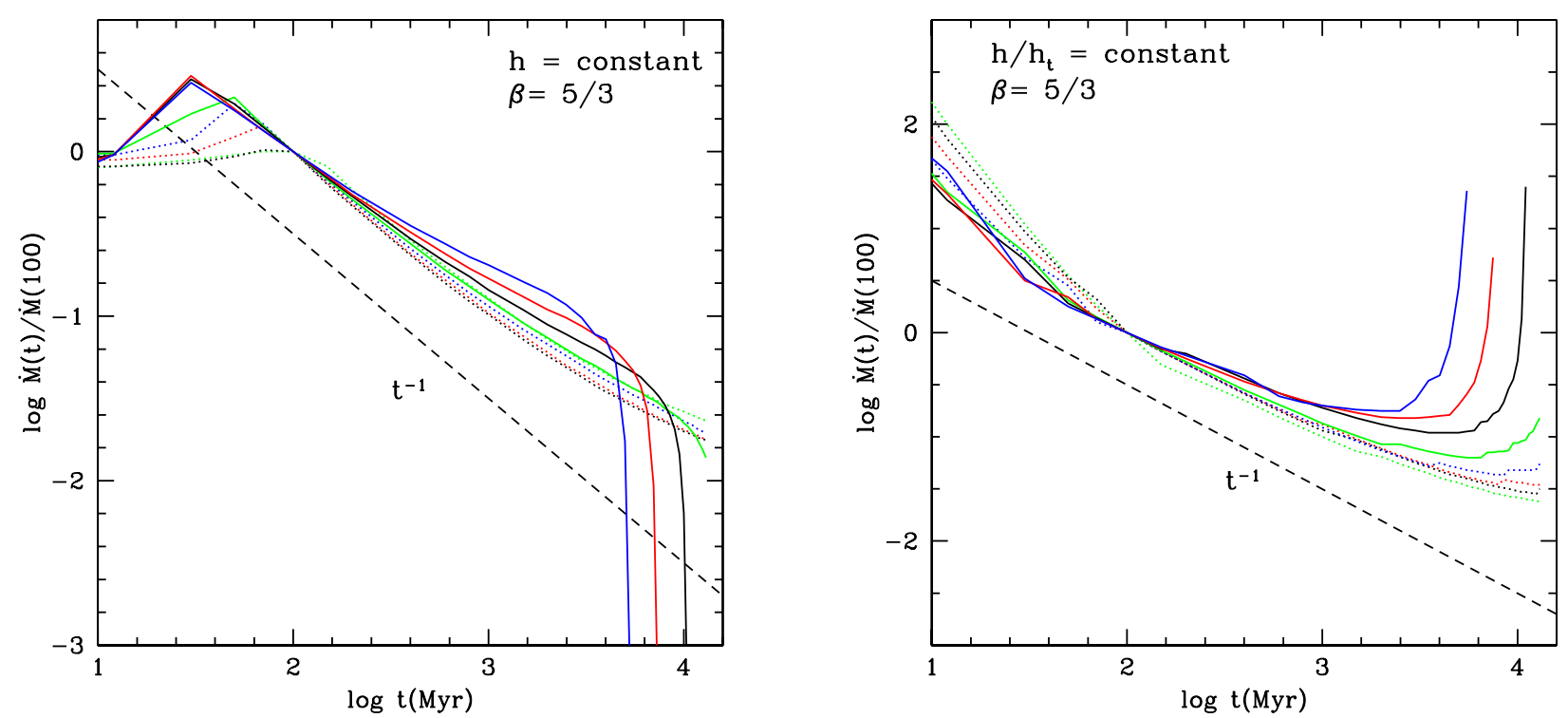

Fig. 10. Ratio of the mass loss rate at time $t, \dot{M}(t)$, with respect to the mass loss rate at $t=100 \mathrm{Myr}, \dot{M}(100)$. Dotted lines are for $M / M_{\odot}=0.5$ (green), 0.6 (black), 0.7 (red) and 0.8 (blue). Continuous lines are for $M / M_{\odot}=0.9$ (green), 1.0 (black), 1.1 (red) and 1.2 (blue). As indicated, the black dashed line shows the $1 / t$ function, which is a good approximation to the mass loss rate when the moment of inertia is constant and the evolution of the angular momentum is described by an inverse square root law. The color figure can be viewed online.

$100 \mathrm{Myr}$ and decrease very rapidly as the star approaches the end of main sequence. Obviously, this is mainly driven by changes in the moment of inertia. Intuitively, this does not make sense. The mass loss rate is expected to decrease as the star enters the main sequence since, among other things, the surface area will shrink and the escape velocity will increase as the star contracts. On the contrary, towards the end of main sequence the mass loss rate should tend to increase since the area will be larger and the escape velocity smaller as the star expands. Thus, a constant physical depth for the magnetic sources leads to an unlikely scenario.

(iii) If the physical depth of magnetic field sources relative to the physical depth of the convective envelope, $h / h_{t}$, is constant in time, mass loss rates of all spectral types decrease during the first $100 \mathrm{Myr}$ and increase during the second half of their main sequence. This is mainly driven by changes in the moment of inertia and depth of the convective envelope. Since this is a more likely scenario, it follows that this is a better case for this magnetic torque model, as long as the empirical formula for the torque holds its ground for stars younger than the Pleiades cluster.

In either one of these scenarios ( $h$ or $h / h_{t}$ constant in time) the mass dependent function, $A(M)$, can be calibrated using a star with known mass, age and mass loss rate. At present, these quantities are known with any certainty for only one star, the Sun. Assuming that it did not have a unique rotational history, the magnetic torque model where $h / h_{t}$ is constant implies that the present solar mass loss rate is very close to its lowest value (2 - $3 \times 10^{-14} M_{\odot} \mathrm{yr}^{-1}$, Wang 1998) and, if magnetic braking is still dominant, leads to a mass loss rate that may be $5-8 \times 10^{-14} M_{\odot} \mathrm{yr}^{-1}$ when our star is $9000 \mathrm{Myr}$ old. Under this scenario, the mass loss rate may have been 2 to $3 \times 10^{-13} M_{\odot} \mathrm{yr}^{-1}$ when the Sun was around 100 Myr old, if its rotational period was similar to the rotational period of solar type stars in the Pleiades cluster (as discussed in $\S 3.1$, this is not unlikely).

Mass loss rates of a handful of late type main and post-main sequence stars have been calculated from astrospheric absorption in the stellar Lyman- $\alpha$ emission line (Wood et al. 2005; Linsky \& Wood 2014), 


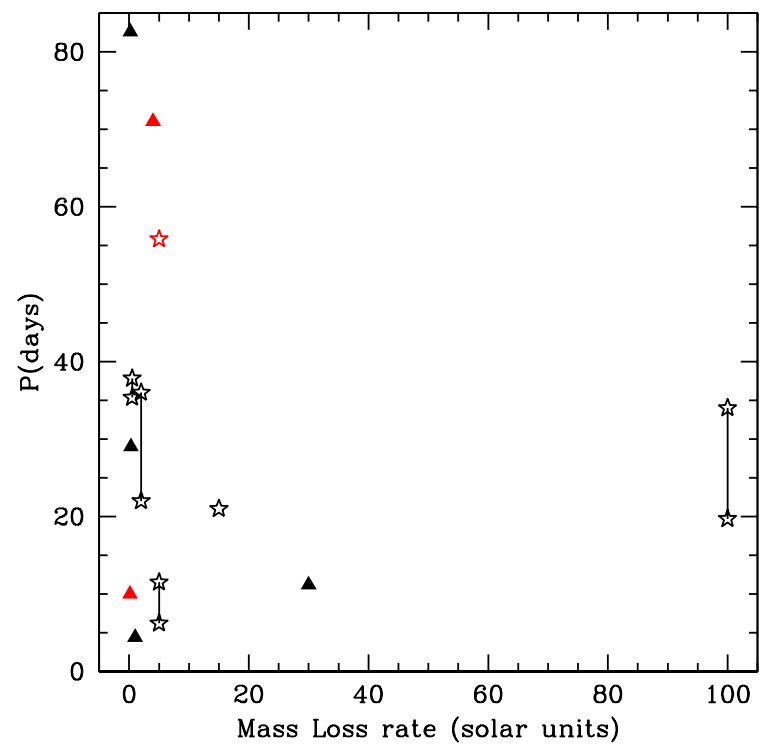

Fig. 11. Rotational periods as a function of mass loss rates. Main sequence stars in black, post-main sequence stars in red. Triangles stand for single star measurements and starred symbols (linked by a line when more than one period has been measured) for measurements including both stars. All mass loss rates are taken from Wood et al. (2005). Rotational periods stem from a variety of sources: Proxima Cen (Collins, Jones \& Barnes 2017), $\alpha$ Cen A (Bazot et al. 2007), $\alpha$ Cen B (Dewarf, Datin \& Guinan 2010), $\epsilon$ Eri (Frölich 2007), 61 Cyg A, 36 Oph A and B, 70 Oph A and B, 61 Vir and $\delta$ Eri (Baliunas, Sokoloff \& Soon 1996), $\lambda$ And (Kukarkin et al. 1971), EV Lac (Contadakis 1995), $\xi$ Boo A and B (Noyes et al. 1984) and DK UMa (Gondoin 2005). The color figure can be viewed online.

the only available technique to measure this quantity. There is a good looking correlation between mass loss rate and X-ray flux, as long as the latter is smaller than $\approx 10^{6} \mathrm{erg} \mathrm{cm}^{-2} \mathrm{~s}^{-1}$. Combining this correlation with an X-ray flux vs. age relationship (Ayres 1997), Wood et al. (2005) affirm that $\dot{M} \alpha t^{-2.33 \pm 0.55}$ in stars where the X-ray flux is smaller than the aforementioned limit. Except for stars younger than $\approx 100 \mathrm{Myr}$, this relationship is at odds with all the estimates that have just been mentioned.

Different explanations can be tried to account for these discrepancies. But the problem may be directly associated to the mass loss rate vs. time relationship put forward by Wood et al. (2005), since there is no connection between the mass loss rate and the rotational period of these stars, as can be seen in Figure 11. Under this circumstance, it is unlikely that a relation between mass loss rate and age is hidden in their data set. This result should not be unexpected, since the mass loss rate data set stems from a very diverse group of single and binary stars: giant, sub-giant and G2 to M5.5 dwarf stars. Furthermore, there is no way to know the individual mass loss rates in the binary systems $\alpha$ Cen, $36 \mathrm{Oph}, \lambda$ And, $70 \mathrm{Oph}$ and $\xi \mathrm{Boo}$, since the size of the modeled astrosphere includes both stars. More measurements of the mass loss rate of late type main sequence stars are required to have a better understanding of this quantity as a function of mass and age. An important addition to this discussion is the effect that coronal mass ejections may have on the mass loss rate and rotational braking (e.g., Cranmer 2017).

\section{CONCLUSIONS}

A database comprising the rotational period, $2 M A S S$ photometry and Gaia parallax and photometry of thousands of late type main sequence stars was put together. These stars were collected from the CoRoT satellite, the HATNet survey, the Kepler satellite field and 17 open clusters comprising a 13 to $3500 \mathrm{Myr}$ age range. Special care was taken to exclude stars with a precision worse than $20 \%$ in their Gaia parallax and photometry and $2 M A S S$ photometry, that are or may be part of a multiple system, and that have light curve modulations produced by other sources of variability besides rotation. Stellar parameters were deduced matching their absolute magnitudes with those predicted by different sets of YaPSI stellar isochrones, in order to analyze the behavior of angular momentum and other physical quantities as a function of mass and age.

In a figure displaying rotational periods as a function of mass (Figure 2), the vast majority of Kepler field main sequence low mass stars lie within a region with clearly defined lower and upper limits (short and long periods). The lower boundary traces the rotational period of stars once they have stabilized in the main sequence. It is also traced by slowly rotating Pleiades stars. The M 35 cluster is supposed to be $\approx 35$ Myr older than the Pleiades. The location of its slow rotators runs in parallel but with slightly shorter periods, suggesting that M 35 is younger than the Pleiades.

Very few fast rotating $0.5-0.8 M_{\odot}$ main sequence stars are present in Praesepe (750 Myr), and none are found in NGC 6811 (1000 Myr) and NGC 752 (1350 Myr). Thus, nearly all $0.5-0.8 M_{\odot}$ stars merge into a single slowly rotating mass dependent 
sequence after $\approx 750 \mathrm{Myr}$. No fast $0.8-1.2 M_{\odot}$ rotators were found in M 48 (500 Myr), M 37 (450 Myr) and the Pleiades (120 Myr), a few in M 35 (155 Myr, probably less) and some more in M 34 (210 Myr). Thus, most $0.8-1.2 M_{\odot}$ main sequence stars seem to merge into a single slowly rotating sequence after $\approx 120 \mathrm{Myr}$. This implies that rotational convergence in the high mass range happens earlier than previously thought (around $600 \mathrm{Myr}$ ).

If stars rotate as rigid bodies - more precisely, if there is no gradient in the radial average of the angular velocity - the angular momentum of the vast majority of 0.5 to $1.2 M_{\odot}$ stars is confined within clearly outlined upper and lower bounds. The lower boundary is defined by a couple of equations. One is valid for $0.9 \leq M / M_{\odot} \leq 1.2$, and the age of this stars is taken as nearly equal to their main sequence lifetime. The second equation applies to less massive stars, with an assumed age that is close to the Milky Way's. It can be said that these equations define a terminal rotational main sequence isochrone. The upper boundary coincides with the position occupied by the slowest rotators of the Pleiades cluster, thus defining a zero age rotational main sequence at $\approx 120$ Myr. Stars from the Praesepe cluster delineate a third rotational isochrone at $\approx 750 \mathrm{Myr}$. The difference between these isochrones is increasingly smaller for less massive stars, showing that the angular momentum loss rate during main sequence is less efficient in low mass stars.

Three types of relationships between angular momentum and age were compared with the average and minimum values of angular momenta of stars within open clusters with known age, as well as the angular momentum of the Sun and stars with ages derived form asteroseismological data modeling. A simple exponential relation, calibrated with the Pleiades and Praesepe isochrones, is at odds with the data. A "classical" inverse square root relation implies that the shape of the angular momentum vs. mass distribution is time invariant, but this is not observed. Calibrated with the Praesepe isochrone, this relationship does not hold for stars younger than $\approx 500 \mathrm{Myr}$, but is consistent with older stars that are more massive than $0.7 M_{\odot}$. A better fit throughout the entire age range is an exponential solution involving the three isochrones and three mass dependent parameters.

During the six hundred million years separating the Pleiades and Praesepe isochrones, the mean angular momentum loss rate is around 90 and a bit more than $40 \%$ per Gyr for 1.2 and $0.5 M_{\odot}$ stars. Afterwards, loss rates are much smaller and near the end of main sequence $\approx 87 \%$ of the initial angular momentum of 0.9 to $1.2 M_{\odot}$ stars has been lost. At this point in time, the oldest 0.7 and $0.5 M_{\odot}$ stars have lost $80 \%$ and $73 \%$ of the angular momentum they had when they set foot on the Pleiades isochrone.

Age estimates based on the angular momentum are likely to be reasonable for middle aged stars if $M \geq 0.7$. These estimates are unreliable at early times, since there is a wide range of periods in contemporary $\mathrm{F}$ and $\mathrm{G}$ stars younger than $450 \mathrm{Myr}$ and $750 \mathrm{Myr}$ when $0.6 \leq M / M_{\odot} \leq 0.7$. This is also true in aging stars, since rotational periods and angular momenta tend to accumulate in a decreasing range of values, particularly for lower mass stars.

An inverse square root relation between rotational period and age was found to be valid only after $\approx 500-750 \mathrm{Myr}$ and as long as there is no significant change in the moment of inertia, i.e., roughly up to to the end of the first half of the main sequence (1400, 3600,4500 and $7900 \mathrm{Myr}$ if $M / M_{\odot}=1.2,1.1,1.0$, and 0.9 ). The data indicate that during the second half of the main sequence the evolution of the rotational period is increasingly being determined by changes in the moment of inertia, less so by other agents of rotational braking.

The evolution of the Rossby number was computed using the exponential and inverse square root approximations to the evolution of the angular momentum, in combination with YaPSI convective turnover times from isochrones with $[\mathrm{Fe} / \mathrm{H}]=0.0$ and $Y_{0}=0.28$. It turns out that it is larger than one for 1.2, 1.1, 1.0 and $0.9 M_{\odot}$ stars, once they are older than $\approx 500,2500,5500$ and 9500 Myr. This implies that the Parker dynamo mechanism may cease before there is a significant change in the moment of inertia of 1.2 and $1.1 M_{\odot}$ stars. If so, their surviving magnetic fossil field, other kind of dynamos such as the Babcock-Leighton cycle - or local magnetic fields produced by turbulent plasmas, may be the main rotational braking source during the time interval between the interruption of the Parker dynamo and the onset of the phase where the moment of inertia grows in a significant way. Later on, the transformation of the moment of inertia determines the evolution of the rotational period. In less massive stars, dynamo activity will decrease at the same time as the moment of inertia escalates.

The exponential and inverse square root approximations to the evolution of the angular momentum were also used to obtain an empirical formula for the torque, which is very powerful and variable at least during the first $\approx 500$ Myr. Thus, the magnetic 
field strength and/or the mass loss rate is extremely robust and mutable during the early stages. Later on, after dropping by a couple of orders of magnitude, the torque and at least one of these agents must be increasingly weaker and stable. When this is so, the torques derived from the exponential and inverse square root approximations are nearly identical.

An idealized model of the torque based on these empirical approximations, on observed relationships between the rotational period and the superficial magnetic field (Saar 1996; Vidotto et al. 2014), and on the assumption that the magnetic field is produced by identical dipoles located at a physical depth that is constant in time with respect to the width of the convective envelope, leads to an uncalibrated mass and time dependent equation for the mass loss rate of late type stars. According to this model, after $100 \mathrm{Myr}$ the mass loss rate functions of all 0.5 - $1.2 M_{\odot}$ stars converge to a time dependence that is roughly proportional to $t^{-1}$. Towards the end of the main sequence, the mass loss rate is swiftly taken back to its initial value. If the rotational period of the Sun was near the Pleiades isochrone when it was $\approx 100 \mathrm{Myr}$ old, its mass loss rate may have been close to $3 \times 10^{-13} M_{\odot} \mathrm{yr}^{-1}$. If the magnetic torque is still dominant, it will have a mass loss rate between 5 and $8 \times 10^{-14} M_{\odot} \mathrm{yr}^{-1}$ when it hits the $9000 \mathrm{Myr}$ mark. If this model is reasonably close to reality, the mass loss rate was about five times more vigorous when life on Earth started $4280 \mathrm{Myr}$ ago (Dodd et al. 2017).

The author acknowledges an unknown referee for a rigorous and productive revision.

\section{APPENDIX \\ A. DATA SOURCES FOR CLUSTER PARAMETERS}

h Persei (Melotte 13; NGC 869). Cluster coordinates and angular radius, Kharchenko et al. (2005, henceforth Ka05); parallax, here; age, Mayne (2008) and Currie et al. (2010); color excess, Ka05 and Currie et al. (2010); $[\mathrm{Fe} / \mathrm{H}]$ (unknown). The cluster parallax is the mean Gaia DR2 parallax of 203 stars from Moraux et al. (2013); stellar parallaxes where the difference with the mean is greater than 0.227 mas were rejected. The mean parallax implies a distance of $1830 \pm 400 \mathrm{pc}$, which is $20 \%$ smaller than other estimates (Slesnick, Hillenbrand \& Masey 2002; Mayne \& Naylor 2008; Currie et al. 2010).

IC 4665 (Collinder 349; Melotte179). Coordinates and parallax, Babusiaux et al. (2018, hence- forth Ba18); angular radius, Ka05; age, Ka05, Manzi et al. (2008), Cargile \& James (2010) and Nepotil et al. (2016, henceforth Ne16); color excess, Mermilliod (1981) and Dias et al. (2002, henceforth Di02); $[\mathrm{Fe} / \mathrm{H}]$, Ne16. There is a very small difference between the distance implied by the Gaia DR2 parallax, $346 \mathrm{pc}$, and previous determinations (Ka05; Cargile \& James 2010).

NGC 2547. Coordinates and parallax, Ba18; angular radius, Ka05; age, Ka05 and Ne16; color excess, Clariá (1982), Naylor \& Jeffries (2006), Paunzen et al. (2014) and Ba18; $[\mathrm{Fe} / \mathrm{H}]$, Ne16. There is $\mathrm{a} \approx \pm 10 \%$ difference between the inferred distance from the Gaia DR2 parallax, 393 pc, and other estimates (Ka05; Naylor \& Jeffries 2006).

Blanco 1. Coordinates, Platais et al. (2011); angular radius, Ka05; parallax, here; age, Ne16, Cargile \& James (2010) and Juárez et al. (2014); color excess, Di02 and Ba18; $[\mathrm{Fe} / \mathrm{H}]$, Ne16 and Ford, Jeffries \& Smalley (2005). The cluster parallax is the mean Gaia DR2 parallax of 22 stars from Cargile et al. (2014); stellar parallaxes where the difference with the mean is greater than 0.080 mas were not considered. The mean parallax implies a distance of $238 \pm 2$ pc to Blanco 1, almost identical to other estimates (Ka05; van Leeuwen 2009, henceforth vL09; Ba18).

Pleiades (M 45; Melotte 22). Coordinates and angular radius, Ka05; parallax, Ba18; age, Basri \& Marcy (1996), Barrado y Navascués et al. (2004), Ka05, vL09, Dahm (2015) and Ba18; color excess, O'Dell, Hendry \& Collier Cameron (1994), Di02 and Ba18; $[\mathrm{Fe} / \mathrm{H}]$, Ne16. With the exception of vL09, there is a very small difference between previous distance estimates and the one inferred from the Gaia DR2 parallax, $136 \mathrm{pc}$.

M 35 (NGC 2168). Coordinates and parallax, Ba18; angular radius, Ka05; age, Barrado y Navascués, Deliyannis \& Stauffer (2001), von Hippel et al. (2002), Kalirai et al. (2003), Ka05 and Ne16; color excess, Sung \& Bessell (1999) and Di02; $[\mathrm{Fe} / \mathrm{H}]$, Ne16. The distance found from the Gaia DR2 parallax is $894 \mathrm{pc}$; previous reports from Sung \& Bessell (1999), Kalirai et al. (2003) and McNamara et al. (2011) give a distance of 832, 830, 912 and $732 \mathrm{pc}$ to the cluster.

NGC 2301. Coordinates and angular radius, Ka05; parallax, here; age, Ka05, Ne16 and univie.ac.at/webda/; color excess, univie.ac.at/webda/ and Di02; $[\mathrm{Fe} / \mathrm{H}]$, Ne16. The parallax is the mean Gaia DR2 parallax of 44 stars from the list of Sukhbold \& Howell (2009); stellar parallaxes where the difference with the mean is smaller or greater 
than 0.144 mas were rejected. The mean parallax implies a distance of $975 \pm 80$ pc to NGC 752 or $\approx 10 \%$ larger than other reported values (Ka05; univie.ac.at/webda/).

M 34 (NGC 1039). Coordinates and parallax, Ba18; angular radius, Ka05; age, Ianna \& Schlemmer (1993), Meynet, Mermilliod \& Maeder (1993), Ka05 and Ne16; color excess, Canterna, Crawford \& Perry (1970) and Di02; $[\mathrm{Fe} / \mathrm{H}], \mathrm{Ne} 16$. The distance found from the Gaia DR2 parallax, $512 \mathrm{pc}$, is $\approx 5 \%$ larger than previous calculations (Jones \& Prosser 1996; $\mathrm{Ka} 05)$.

M 37 (NGC 2099). Coordinates and angular radius, Ka05; parallax, here; age, Nilakshi \& Sagar (2002), Ka05, Hartman et al. (2008) and Pancino et al. (2010); color excess, Di02; Nilakshi \& Sagar (2002) and Pancino et al. (2010); [Fe/H], Hartman et al. (2008), Pancino et al. (2010) and Ne16. The cluster parallax is the mean Gaia DR2 parallax of 309 stars from the list of Nuñez et al. (2015); stellar parallaxes where the difference with the mean is smaller or greater than 0.149 mas were rejected. The mean parallax implies a distance of $1420 \pm 145 \mathrm{pc}$ to M 37, nearly equal to other estimates (Kalirai et al. 2001a; Nilakshi \& Sagar 2002; Ka05; Hartman et al. 2008).

M 48 (NGC 2548). Coordinates, Ka05 and Ba18; angular radius, Ka05; parallax, Ba18; age, BalaguerNúñez et al. (2005), Ka05, Ne16 and Ba18; color excess, Di02, Balaguer-Núñez et al. (2005), Ka05 and Ba18; $[\mathrm{Fe} / \mathrm{H}]$, Di02, Balaguer-Núñez et al. (2005) and Ne16.

Coma Berenices (Melotte 111; Collinder 256). Coordinates and angular radius, Ka05 and vL09; parallax, Ba18; age, Ka05, Silag \& Landstreet (2014), vL09, Ne16 and Ba18; color excess, Nicolet (1981), Di02, Taylor (2006) and Ba18; $[\mathrm{Fe} / \mathrm{H}]$, Ne16. The Gaia DR2 parallax implies a distance of $86 \mathrm{pc}$, a nearly perfect agreement with other estimates (Ka05; vL09; Majaess et al. 2011).

Hyades (Melotte 25; Collinder 50). Coordinates, univie.ac.at/webda/; angular radius, Perryman et al. (1998) with an assumed distance of $47 \mathrm{pc}$; parallax, Ba18; age, Brand \& Huang 2015, Ne16 and Ba18; color excess, Di02, Taylor (2006) and Ba18; $[\mathrm{Fe} / \mathrm{H}]$, Cummings et al. (2017). The Gaia DR2 parallax implies a distance of $47.6 \mathrm{pc}$ to the cluster, nearly identical to previous estimates (Perryman et al. 1998; vL09; Majaess et al 2011; McArthur et al. 2011).

Praesepe (M 44; NGC 2632). Coordinates and angular radius, Ka05; parallax, Ba18; age, Ka05, Ne16 and Ba18; color excess, Di02 and Taylor (2006);
$[\mathrm{Fe} / \mathrm{H}]$, Ne16 and Cummings et al. (2017). The distance found from the Gaia DR2 parallax is equal to $186 \mathrm{pc}$, almost identical to distances reported by Ka05 and vL09.

NGC 6811 (Melotte 222). Coordinates and angular radius, Ka05; parallax, here; age, Janes et al. (2013) and Molenda-Zakowicz et al. (2014); color excess, Janes et al. (2013) and Molenda-Zakowicz et al. (2014); $[\mathrm{Fe} / \mathrm{H}], \mathrm{Ne} 16$. The cluster parallax given in Table 1 is the mean Gaia DR2 parallax of 34 stars from the list of Meibom et al. (2001b); stellar parallaxes where the difference with the mean is smaller or greater than 0.026 mas were rejected. The mean parallax implies a distance of $1145 \pm 20 \mathrm{pc}, 7 \%$ less than other distance reports (Ka05; univie.ac.at/webda/). NGC 752 (Melotte 12). Coordinates, Ka05 and Agüeros et al. (2018); angular radius, Ka05; parallax, here; age, Ka05 and Agüeros et al. (2018); color excess, $\mathrm{Ka} 05 ;[\mathrm{Fe} / \mathrm{H}]$, Ne16. The cluster parallax is the mean Gaia DR2 parallax of 9 stars from the list of Agüeros et al. (2018); stellar parallaxes where the difference with the mean is smaller or greater than 0.333 mas were rejected. The mean parallax implies a distance of $440 \pm 10 \mathrm{pc}$ to NGC 752, nearly equal to other estimates distance (Ka05; Agüeros et al. 2018). NGC 6819 (Melotte 223; Foxhead). Coordinates, Xin \& Deng (2005); angular radius, Platais et al. (2013) and Yang et al. (2013); parallax, here; age, Ne16; color excess, Kalirai et al. (2001b), Di02 and Yang et al. (2013); $[\mathrm{Fe} / \mathrm{H}], \mathrm{Ne} 16$. The cluster parallax is the mean Gaia DR2 parallax of 13 stars from the list of Meibom et al. (2015); stellar parallaxes where the difference with the mean is smaller or greater than 0.043 mas were rejected. The mean parallax implies a distance of $2985 \pm 220$ pc, substantially more $(\approx 12$ to $40 \%)$ than other estimates (Lindoff 1972; Auner 1974; Rosvick \& VandenBerg 1998; Kalirai et al. 2001b).

M 67 (NGC 2682). Coordinates and parallax, Ba18; angular radius, Ka05; age, Ka05 and Ne16; color excess, Montgomery, Marschall \& Janes (1993), Di02 and Ba18; $[\mathrm{Fe} / \mathrm{H}]$, Ne16. There is a small difference between the distance found from the Gaia DR2 parallax and previous estimates (Twarog, Ashman \& Anthony-Twarog 1997; Ka05; Jacobson, Pilachowski \& Friel 2011);

\section{REFERENCES}

Aerts, C., Mathis, S., \& Rogers, T. M. 2019, A\&AR, 57, $35 \mathrm{~A}$

Affer, L., Micela, G., Favata, F., \& Flaccomio, E. 2012, MNRAS, 424, 11

Agüeros, M. A., Bowsher, E. C., Bochanski, J. J., et al. 2018, ApJ, 862, 33 
Agüeros, M. A., Covey, K. R., Lemonias, J. J., et al. 2011, ApJ, 740, 110

Amard, L., Palacios, A., Charbonnel, C., Gallet, F., \& Bouvier, J. 2016, A\&A, 587, A105

Andrae, R., Fouesneau, M., Creevey, O., et al. 2018, A\&A, 616, 8A

Auner, G. 1974, A\&AS, 13, 143

Ayres, T. R. 1997, JGR, 102, 1641

Babusiaux, C., et al. 2018, A\&A, 616, 10G (Ba18)

Balaguer-Núñez, L., Jordi, C., \& Galadí-Enríquez, D. 2005, A\&A, 437, 457

Baliunas, S., Sokoloff, D., \& Soon, W. 1996, ApJ, 457, L99

Baraffe, I., Homeier, D., Allard, F., \& Chabrier, G. 2015, A\&A, 577, A42

Barnes, J. R., Collier Cameron, A., Donati, J.-F., et al. 2005, MNRAS, 357, L1

Barnes, S. A. 2007, ApJ, 669, 1167

Barnes, S. A., Weingrill, J., Fritzewski, D., Strassmeier, K. G., \& Platais, I. 2016, ApJ, 823, 16

Barrado y Navascués, D., Deliyannis, C. P., \& Stauffer, J. R. 2001, ApJ, 549, 452

Barrado y Navascués, D., Stauffer, J. R., \& Jayawardhana, R. 2004, ApJ, 614, 386

Basri, G., Marcy, G. W., \& Graham, J. R. 1996, ApJ, 458, 600

Bazot, M., Bouchy, F., Kjeldsen, H., et al. 2007, A\&A, 470, 295

Benomar, O., Takata, M., Shibahashi, H., Ceillier, T., \& García, R. A. 2015, MNRAS, 452, 2654

Bohigas, J., Carrasco, L., Torres, C. A. O., \& Quast, G. R. 1986, A\&A, 157, 278

Bouvier, J., Cabrit, S., Fernández, M., Martin, E. L., \& Mathews, J. M. 1993, A\&A, 272, 176

Brandt, T. D. \& Huang, C. X. 2015, ApJ, 807, 58

Brown, B. P., Browning, M. K., Brun, A. S., Miesch, M. S., \& Toomre, J. 2010, ApJ, 711, 424

Brown, T. M., Christensen-Dalsgaard, J., Dziembowski, W. A., et al. 1989, ApJ, 343, 526

Brown, T. M., Latham, D. W., Everett, M. E., \& Esquerdo, G. A. 2011, AJ, 142, 112

Bumba, V. \& Howard, R. 1965, ApJ, 141, 1492

Busse, F. H. 1970, ApJ, 159, 629

Canterna, R., Crawford, D. L., \& Perry, C. L. 1970, PASP, 91, 541

Cargile, P. A. \& James, D. J. 2010, AJ, 140, 677

Cargile, P. A., James, D. J., Pepper, J., et al. 2014, ApJ, 782,29

Carpenter, J. M. 2001, AJ, 121, 2851

Ceillier, T., van Saders, J., García, R. A., et al. 2016, MNRAS, 456, 119

Chaboyer, B., Demarque, P., \& Pinnsonneault, M. H. 1995, ApJ, 441, 865

Charbonneau, P. \& MacGregor, K. B. 1993, ApJ, 417, 762

Charbonneau, P. 2010, LRSP, 7, 3

Charbonnel, C. \& Talon, S. 2005, Science, 309, 2189
Charbonnel, C., Decressin, T., Amard, L., Palacios, A., \& Talon, S. 2013, A\&A, 554, A40

Choi, J., Dotter, A., Conroy, C., et al. 2016, ApJ, 823, 102

Clariá, J. J. 1982, A\&AS, 47, 323

Collier Cameron, A. \& Campbell, C. G. 1993, A\&A, 274, 309

Collier Cameron, A., Campbell, C. G., \& Quaintrell, H. 1995, A\&A, 298, 133

Collier Cameron, A., Davidson, V. A., Hebb, L., et al. 2009, MNRAS, 400, 451

Collins, J. M., Jones, H. R. A., \& Barnes, J. R. 2017, A\&A, 602, A48

Covey, K. R., Agüeros, M. A., Law, N. M., et al. 2016, ApJ, 822, 81

Contadakis, M. E. 1995, A\&A, 300, 819

Cranmer, S. R., van Ballegooijen, A. A., \& Edgar, R. J. 2007, ApJS171 520

Cranmer, S. R. \& Saar, S. H. 2011, ApJ, 741, 54

Cranmer, S. R. 2017, ApJ, 840, 114

Cummings, J. D., Deliyannis, C. P., Maderak, R. M., \& Steinhauer, A. 2017, AJ, 153, 128

Currie, T., Hernández, J., Irwin, J., et al. 2010, ApJS, 186, 191

Dahm, S. E. 2015, ApJ, 813, 108

Dartmouth, F. G., Chaboyer, B., \& Dotter, A. 2011, ApJ, 740, L25

Davies, G. R. et al. 2015, MNRAS, 446, 2259

Delorme, P., Collier Cameron, A., Hebb, L., et al. 2011, MNRAS, 413, 2218

Denissenkov, P. A., Pinsonneault, M., Terndrup, D. M., \& Newsham, G. 2010, ApJ, 716, 1269

Denissenkov, P.A. 2010, ApJ, 719, 28

DeWarf, L., Datin, K., \& Guinan, E. 2010, ApJ, 722, 343

Dias, W. S., Alessi, B. S., Moitinho, A. \& Lépine, J. R. D. 2002, A\&A, 389, 871 (Di02)

Dodd, M. S., Papineau, D., Grenne, T., et al. 2017, Natur, 543, 60

Douglas, S. T., Agüeros, M. A., Covey, K. R., et al. 2016, ApJ, 822, 47

Donati, J.-F. et al. 1992, A\&A, 265, 682

Donati, J.-F. \& Collier Cameron, A. 1997, MNRAS, 291, 1

Durney, B. 1970, ApJ, 161, 1115

Durney, B. R. \& Latour, J. 1978, GApFD, 9, 241

Durney, B. R., De Young, D. S., \& Roxburgh, I. W. 1993, SoPh, 145, 207

Eff-Darwich, A. \& Korzennik, S. G. 2013, SoPh, 287, 43

Epstein, C. R. \& Pinsonneault, M. H. 2014, ApJ, 780, 159

Evans, D. W., Riello, M., DeAngeli, F., et al. 2018, A\&A, 616, A4

Finley, A. J. \& Matt, S. P. 2018, ApJ, 854, 78

Ford, A., Jeffries, E. D., \& Smalley, B. 2005, MNRAS, 364,272

Fröhlich, H.-E. 2007, AN, 328, 1037 
Gebran, M., Monier, R., \& Richard, O. 2008, A\&A, 479, 189

Gallet, F. \& Bouvier, J. 2013, A\&A, 556, A36 2015, A\&A, 577, A98

Gondoin, P. 2005, A\&A, 444, 531

Han, E., Wang, S. X., Wright, J. T., et al. 2014, PASP, 126,827

Hartman, J. D., Gaudi, B. S., Holman, M. J., et al. 2008, ApJ, 675, 1233

Hartman, J. D., Gaudi, B. S., Pinsonneault, M. H., et al. 2009, ApJ, 691, 342

Hartman, J. D., Bakos, G. A., Kovács, G., \& Noyes, R. W. 2010, MNRAS, 408, 475

Hartman, J. D., Bakos, G. A., Noyes, R. W., et al. 2011, ApJ, 141, 166

Henderson, C. B. \& Stassun, K. G. 2012, ApJ, 747, 51

Henry, G. W., Eaton, J. A., Hamer, J., \& Hall, D. S. 1995, ApJS, 97, 513

Herbig, G. \& Spalding, J. F. 1955, ApJ, 121, 118

Herbst, W., Bailer-Jones, C. A. L., Mundt, R., Meisenheimer, K., \& Wackermann, R. 2002, A\&A, 396, 513

Holl, et al. 2018, A\&A, 618, A30

Ianna, P. A. \& Schlemmer, D. M. 1993, AJ, 105, 209

Irwin, J., Aigrain, S., Hodgkin, S., et al. 2006, MNRAS, 370,954

Irwin, J., Hodgkin, S., Aigrain, S., et al. 2008, MNRAS, 383,1588

Indebetouw, R., et al. 2005, ApJ, 619, 931

Jacobson, H. R., Pilachowski, C. A., \& Friel, E. D. 2011, AJ, 142,59

James, D. J., Barnes, S. A., Meibom, S., et al. 2010, $\mathrm{A} \& \mathrm{~A}, 515, \mathrm{~A} 100$

Janes, K, Barnes, S. A., Meibom, S., \& Hoq, S. 2013, AJ, 145,7

Johnstone, C. P., Güdel, M., Brott, I., \& Lüfinger, T. 2015a, A\&A, 577, A28

Johnstone, C. P., Güdel, M., Lüfinger, T., Toth, G., \& Brott, I. 2015b, A\&A, 577, A27

Johnstone, C. P., Pilat-Lohinger, E., Lüfinger, T., Güdel, M., \& Stöcl, A. 2019, A\&A, 626, A22

Jones, B. F. \& Prosser, C. F. 1996, AJ, 111, 1193

Juárez, A. J., Cargile, P. A., James, D. J., \& Stassun, K. G. 2014, ApJ, 795, 143

Kalirai, J. S., Ventura, P., Richer, H. B., et al. 2001a, AJ, 122, 3239

Kalirai, J. S., Richer, H. B., Fahlman, G. G., et al. 2001b, AJ, 122, 266

Kalirai, J. S., Fahlman, G. G., Richer, H. B., \& Ventura, P. 2003 AJ, 126, 1402

Kawaler, S. D. 1988, ApJ, 333, 236

Kharchenko, N. V., Piskunov, A. E., Roeser, S., Schilbach, E., \& Scholz, R.-D. 2005, A\&A, 438, 1163 (Ka05)

Kitchatinov, L. L. \& Rüdiger, G. 2005, AN, 326, 379

Kitchatinov, L. L. \& Nepomnyashchikh, A. 2017, MNRAS, 470, 3124

Koenigl, A. 1991, ApJ, 370, L39

Koppen, J. \& Vergely, J.-L. 1998, MNRAS, 299, 567
Korzennik, S. G. \& Eff-Darwich, A. 2012, PASPC, 462, 267

Kraft, R. P. 1967, ApJ, 150, 551

Küker, M. \& Rüdiger, G. 2008, JPhCS, 118, 2029

Kukarkin, B. V., Kholopov, P. N., Pskovsky, Y. P., et al. 1971, General catalog of Variable Stars, 3rd ed.

Landin, N. R., Mendes, L. T. S., \& Vaz, L. P. R. 2010, A\&A, 510, A46

Lang, P., Jardine, M., Morin, J., et al. 2014, MNRAS, 439, 2122

Lindoff, U. 1972, A\&AS, 7, 497

Linsky, J. L. \& Wood, B. E. 2014, ASTRP, 1, 43

Lomb, N. R. 1976, Ap\&SS, 39, 447

Lund, M. N., Miesch, M. S., \& Christensen-Dalsgard, J. 2014, ApJ, 790, 121

MacGregor, K. B. \& Brenner, M. 1991, ApJ, 376, 204

Mädler, T., Jofre, P., Gilmore, G., et al. 2016, A\&A, 595, A59

Majaess, D. J., Turner, D. G., Lane, D. J., \& Krajci, T. 2011, JAVSO, 39, 219

Mamajek, E. E. \& Hillenbrand, L. A. 2008, ApJ, 687, 1264

Manzi, S., Randich, S., de Wit, W. J., \& Palla, F. 2008, A\&A, 479, 141

Marigo, P. et al. 2017, ApJ, 835, 77

Marshal, D. J., Robin, A. C., Reylé, C., Schulteis, M., \& Picaud, S. 2006, A\&A, 453, 635

Mathur, S., Metcalfe, T. S., Woitaszek, M., et al. 2012, ApJ, 749, 152

Matt, S. P., Brun, A. S., Baraffe, I., Bouvier, J., \& Chabrier, G. 2015, ApJ, 799, L23

Mayne, N. J. \& Naylor, T. 2008, MNRAS, 386, 261

McArthur, B. E., Benedict, G. F., Harrison, T. E., \& van Altena, W. 2011, AJ, 141, 172

McComas, D. J., Barraclough, B. L., Funsten, H. O., et al. 2000, JGR, 105, 10419

McNamara, B. J., Harrison, T. E., McArthur, B. E., \& Benedict, G. F. 2011, AJ, 142, 53

McQuillan, A., Aigrain, S., \& Mazeh, T. 2103, MNRAS, 432,1203

McQuillan, A., Mazeh, T., \& Aigrain, S. 2014, ApJS, 211,24

Meibom, S., Mathieu, R. D., \& Stassun, K. G. 2009, ApJ, 695,679

Meibom, S., Mathieu, R. D., Stassun, K. G., Liebesny, P., \& Saar, S. H. 2011a, ApJ, 733, 115

Meibom, S., et al. 2011b, ApJ, 733, L9

Meibom, S., Barnes S. A., Platais, I., et al. 2015, Natur, 517,589

Melis, C., Reid, M. J., Mioduszewski, A. J., Stauffer, J. R., \& Bower, G. C. 2014, Science, 345, 1029

Mermilliod, J. C. 1981, A\&A, 97, 235

Mestel, L. 1968, MNRAS, 138, 359

Mestel, L. \& Spruit, H. C. 1987, MNRAS, 226, 57

Mestel, L. \& Weiss, N. O. 1987, MNRAS, 226, 123

Metcalfe, et al. 2014, ApJS, 214, 27

Meynet, G., Mermilliod, J., \& Maeder, A. 1993, A\&AS, 98,477 
Molenda-Zakowicz, J., Brogaard, K., Niemczura, E., et al. 2014, MNRAS, 445, 2446

Montgomery, K. A., Marschall, L. A., \& Janes, K. A. 1993, AJ, 106, 181

Moraux, E., Artemenko, S., Bouvier, J., et al. 2013, $\mathrm{A} \& \mathrm{~A}, 560, \mathrm{~A} 134$

Morin, J., Donati, J.-F., Petit, P., et al. 2010, MNRAS, 407, 2269

Naylor, T. \& Jeffries, R. 2006, MNRAS, 373, 1251

Nepotil, M., Paunzen, E., Heiter, U., \& Soubiran, C. 2016, A\&A, 585, A150 (Ne16)

Newton, E. R., Mondrik, N., Irwin, J., Winters, J. G., \& Charbonneau D. 2018, AJ, 156, 217

Nicolet, B. 1981, A\&A, 104, 185

Nielsen, M. B., Gizon, L., Schunker, H., \& Karoff, C. 2013, A\&A, 557, L10

Nielsen, M. B., Schunker, H., Schou, J., \& Ball, W. H. 2017, A\&A, 603, A6

Nilakshi \& Sagar, R. 2002, A\&A, 381, 65

Noyes, R. W., Hartmann, L. W., Baliunas, S. L., Duncan, D. K., \& Vaughan, A. H. 1984, ApJ, 279, 763

Nuñez, A., Agüeros, M. A., Covey, K. R., et al. 2015, ApJ, ,, 809, 161

Ochsenbein, F., Bauer, P., \& Marcout, J. 2000, A\&AS, 143,23

O'dell, M. A., Hendry, M. A., \& Collier Cameron, A. 1994, MNRAS, 268, 181

Pallavicini, R., Golub, L., Rosner, R., et al. 1981, ApJ, 248,279

Pancino, E., Carrera, R., Rossetti, E., \& Gallart, C. 2010, A\&A, 511, A56

Parker, E. N. 1955, ApJ, 122, 293

1958, ApJ, 128, 664

1988, ApJ, 330, 474

Paunzen, E., Netopil, M., Maitzen, H. M., et al. 2014, $\mathrm{A} \& \mathrm{~A}, 564, \mathrm{~A} 42$

Perryman, M. A. C., Brown, A. G. A., Lebreton, Y., et al. 1998, A\&A, 331, 81

Pizzolato, N., Maggio, A., Micela, G., Sciortino, S., \& Ventura, P. 2003, A\&A, 397, 147

Platais, I., Girard, T. M., Vieira, K., et al. 2011, MNRAS, 413, 1024

Platais, I., Gosnell, N. M., Meibom, S., et al. 2013, AJ, 146,43

Pourbaix, D., Tokovinin, A. A., Batten, A. H., et al. 2004, A\&A, 424, 727

Press, W. H. \& Rybicki G. B. 1989, ApJ, 338, 277

Radick, R. R, Thompson, D. T., Lockwood, G. W., Duncan, D. K., \& Baggett, W. E. 1987, ApJ, 321, 459

Radick, R. R, Lockwood, G. W., Skiff, B. A., \& Thompson, D. T. 1995, ApJ, 452, 332

Rebull, L. M., Wolff, S. C., \& Strom, S. E. 2004, AJ, 127,1029

Rebull, L. M., et al. 2016, AJ, 152, 113

Reiners, A. \& Mohanty, S. 2012, ApJ, 746, 43

Reiners, A., Schüssler, M., \& Passegger, V. M. 2014, ApJ, 794,144
Reinhold, T., Reiners, A., \& Basri, G. 2013, A\&A, 560, A4

Réville, V., Brun, A. S., Matt, S. P., Strugarek, A., \& Brun, A. S. 2015, ApJ, 798, 116

Robrade, J. \& Schmitt, J. H. M. M. 2009, A\&A, 496, 229

Rosvick, J. M. \& VandenBerg, D. 1998, AJ, 115, 1516

Saar, S. H. 1996, IAUS 176, Stellar Surface Structure, ed. K. G. Strassmeier and J. F. Linsky, (Dordrecht), 237

2001, ASPC 223, 11th Cambridge Workshop on Cool Stars, Stellar Systems and the Sun, ed. R. J. García López, R. Rebolo, M. R. Zapatero Osorio, (San Francisco, CA: ASP), 292

Scargle, J. D. 1982, ApJ, 263, 835 1989, ApJ, 343, 874

Schatzman, E. 1962, AnAp, 25, 18

Scholz, A., Eislöffel, J., \& Mundt, R. 2009, MNRAS, 400, 1548

Scholz, A., Irwin, J., Bouvier, J., et al. 2011, MNRAS, 413,2595

Serenelli, A. \& Basu, S. 2010, ApJ, 719, 865

See et al. 2015, MNRAS, 453, 4301

Shulyak, D., Reiners, A., Engeln, A., et al. 2017, NatAs, 1, A184

Shulyak, D. et al. 2019, A\&A, 626, A86

Silag, J. \& Landstreet J.D. 2014, A\&A, 566, A132

Skumanich, A. 1972, ApJ, 171, 565

Slesnick, C. L., Hillenbrand, L. A., \& Masey, P. 2002, ApJ, 576, 880

Slettebak, A. 1955, ApJ, 121, 653

Soderblom, D. R., Jones, B. F., \& Walker, M. F. 1983, ApJ, 274, L37

Soderblom, D. R., Stauffer, J. R., Hudon, J. D., \& Jones, B. F. 1993, ApJS, 85, 315

Spada, F., Demarque, P., Kim, Y.-C., Boyajian, T. S., \& Brewer, J. M. 2017, ApJ, 838, 161 (YaPSI)

Spada, F., Demarque, P., Kim, Y.-C., \& Sills, A. 2013, ApJ, 776, 87

Stauffer, J. R., Hartmann, L. W., Soderblom, D. R., \& Burnham, N. 1984, ApJ, 280, 202

Stauffer, J. R., Hartmann, L. W., Burnham, N., \& Burton, F. F. 1985, ApJ, 289, 247

Struve, O. \& Elvey, C. T. 1931, MNRAS, 91, 663

Sukhbold, T. \& Howell, S. B. 2009, PASP, 121, 1188

Suzuki, T. K., Imada, S., Kataoka, R., et al. 2013, PASJ, 65,98

Taylor, B. J. 2006, AJ, 132, 2453

Thévenin, F., Provost, J., Morel, P., et al. 2002, A\&A, 392, L9

Tomczyk, S., Schou, J., \& Thompson, M. J. 1995, ApJ, 448,57

Tonry, J. L., Stubbs, C. W., Lykke, K. R., et al. 2012, ApJ, ,, 750, 99

Treanor, P. J. 1960, MNRAS, 121, 503

Twarog, B. A., Ashman, K. M., \& Anthony-Twarog, B. J. 1997, AJ, 114, 2556 
Usmanov, A. V., Matthaeus, W. H., Goldstein, M. L., \& Chhiber, R. 2018, ApJ, 865, 25

Vaiana, G. S. et al. 1981, ApJ, 244, 163

VandenBerg, D. A., Bergbusch, P. A., \& Dowler, P. D. 2006, ApJS, 162, 375

van Ballegooijen, A. A., Asgari-Targhi, M., \& Berger, M. A. 2014, ApJ, 787, 87

van der Holst, B., Sokolov, I. V., Meng, X., et al. 2014, ApJ, 782, 81

van Leeuwen, F. 2009, A\&A, 497, 209 (vL09)

van Saders, J. L., Ceillier, T., Metcalfe, T. S., et al. 2016, Natur, 529, 181

Vidotto, A. A., Gregory, S. G., Jardine, M., et al. 2014, MNRAS, 441, 2361

von Hippel, T., Steinhauer, A., Sarajedini, A., \& Deliyan- nis, C. P. 2002, AJ, 124, 1555

Wang, Y.-M. 1998, ASPC 154, The Tenth Cambridge Workshop on Cool Stars, Stellar Systems and the Sun, ed. R. A. Donahue \& J. A. Bookbinder (San Francisco, CA: ASP), 131

Weber, E. J. \& Davis, L. 1967, ApJ, 148, 217

Wenger, M. et al. 2000, A\&AS, 143, 9

Wilson, O. C. 1966, ApJ, 144, 695

Wood, B. E., Redfield, S., Linsky, J. L., Müller, H.-R., \& Zank, G. P. 2005, ApJS, 159, 118

Worthey, G. \& Lee, H-c. 2011, ApJS, 193, 1

Xin, Y. \& Deng, L. 2005, ApJ, 619, 824

Yang, S.-C., Sarajedini, A., Deliyannis, C. P., et al. 2013, ApJ, 762, 3

Joaquín Bohigas: Instituto de Astronomía, Universidad Nacional Autónoma de México, Km. 103 Carretera Tijuana-Ensenada, 22860 Ensenada, Baja California, México (jbb@astro.unam.mx). 